\title{
Rotational spectrum of a chiral amino acid precursor, 2-aminopropionitrile, and searches for it in Sagittarius B2(N) ${ }^{\star \star \star \star}$
}

\author{
H. Møllendal ${ }^{1}$, L. Margulès ${ }^{2}$, A. Belloche ${ }^{3}$, R. A. Motiyenko ${ }^{2}$, A. Konovalov ${ }^{1}$, K. M. Menten ${ }^{3}$, and J. C. Guillemin ${ }^{4}$ \\ ${ }^{1}$ Centre for Theoretical and Computational Chemistry (CTCC), Department of Chemistry, University of Oslo, PO Box 1033 , \\ Blindern, 0315 Oslo, Norway \\ ${ }^{2}$ Laboratoire de Physique des Lasers, Atomes, et Molécules, UMR CNRS 8523, Université de Lille I, 59655 Villeneuve d'Ascq \\ Cedex, France \\ e-mail: laurent.margules@univ-lille1.fr \\ 3 Max-Planck Institut für Radioastronomie, Auf dem Hügel 69, 53121 Bonn, Germany \\ ${ }^{4}$ Sciences Chimiques de Rennes, École Nationale Supérieure de Chimie de Rennes, CNRS, UMR 6226, Avenue du Général Leclerc, \\ CS 50837, 35708 Rennes Cedex 7, France \\ e-mail: jean-claude.guillemin@ensc-rennes.fr
}

Received 6 March 2011 / Accepted 13 October 2011

\begin{abstract}
Context. No chiral molecule has been detected in the interstellar medium (ISM) so far.

Aims. A good candidate for an interstellar detection is 2-aminopropionitrile $\left(\mathrm{CH}_{3} \mathrm{CH}\left(\mathrm{NH}_{2}\right) \mathrm{CN}\right)$, a chiral molecule with a $\mathrm{C}_{3} \mathrm{H}_{6} \mathrm{~N}_{2}$ formula. The first member of this series, aminoacetonitrile $\left(\mathrm{H}_{2} \mathrm{NCH}_{2} \mathrm{CN}\right)$, was recently detected, demonstrating that at least one aminonitrile exists in the ISM.

Methods. Experimental spectra of 2-aminopropionitrile have been recorded in the microwave and submm energy range $(8-80 \mathrm{GHz}$, $150-660 \mathrm{GHz}) . \mathrm{Ab}$ initio calculations facilitated the assignment of the ground state of the most stable conformer and its five lowest excited vibrational states. An unbiased spectral survey of the $80-116 \mathrm{GHz}$ atmospheric window performed with the IRAM $30 \mathrm{~m}$ telescope was used to search for this molecule in the hot core Sgr B2(N). This survey was analyzed in the local thermodynamical equilibrium (LTE) approximation. The emission of 2-aminopropionitrile was modeled simultaneously with the emission of all molecules known in Sgr B2(N), which allowed us to properly take into account line blending and avoid misassignments.

Results. More than 4000 lines of the ground state and the five lowest excited vibrational states (from 185 to $375 \mathrm{~cm}^{-1}$ ) were assigned in the experimental spectrum. The partition function including the vibrational contribution of these states was calculated. Only ten groups of transitions of 2-aminopropionitrile are not severely affected by line blending in the Sgr B2(N) spectrum. Six of them could be considered as tentatively detected but the LTE predictions of the four remaining groups are not (or only marginally) consistent with the observed spectrum. Therefore, only an upper limit of $1.7 \times 10^{16} \mathrm{~cm}^{-2}$ can be securely derived for the column density of 2-aminopropionitrile toward Sgr B2(N), assuming the same source size, temperature, linewidth, and systemic velocity as for aminoacetonitrile

Conclusions. 2-aminopropionitrile is at least 1.8 times less abundant than aminoacetonitrile. A simple comparison to the pair methyl cyanide $\left(\mathrm{CH}_{3} \mathrm{CN}\right)$ /ethyl cyanide $\left(\mathrm{CH}_{3} \mathrm{CH}_{2} \mathrm{CN}\right)$ detected toward $\mathrm{Sgr} \mathrm{B} 2(\mathrm{~N})$ suggests that 2-aminopropionitrile should be 3.5 times less abundant than aminoacetonitrile, which is consistent with the limit derived here.
\end{abstract}

Key words. astrochemistry - ISM: molecules - submillimeter: ISM - line: identification - astronomical databases: miscellaneous ISM: individual objects: Sagittarius B2

\section{Introduction}

It is usually proposed that life on the primitive Earth developed from simple prebiotic organic materials. However, it is more difficult to know where the chemical evolution occured that produced an initial reservoir containing amino acids, sugars, and many other compounds. The early Earth, the pre-solar nebula, or the interstellar medium (ISM) have been proposed independently as the reaction medium. The organic material could accordingly have been produced on Earth starting from simpler

\footnotetext{
* Appendices are available at

http: //www . aanda.org

$\star \star$ Full Table 1 is only available at the CDS via anonymous ftp to cdsarc.u-strasbg.fr $(130.79 .128 .5)$ or via

http://cdsarc.u-strasbg.fr/viz-bin/qcat?J/A+A/538/A51
}

compounds or delivered to Earth by meteorites and comets during the phase of bombardment, and probably both occurred (Joyce et al. 1984; Bailey et al. 1998). The discovery of more and more complex molecules in the ISM supports the hypothesis of an initial material contained in this medium, even if this does not prove the origin of the molecules of the primitive Earth.

About 150 molecules and more than one hundred isotopologues have been detected in the ISM up to date ${ }^{1}$. Simple molecules like acetaldehyde $\left(\mathrm{CH}_{3} \mathrm{CHO}\right)$, hydrogen cyanide $(\mathrm{HCN})$, or ammonia $\left(\mathrm{NH}_{3}\right)$ are some of them but also anions, cations, radicals or inorganic compounds are present. No branched or chiral molecules have been detected. The

\footnotetext{
${ }^{1}$ See, e.g., http://www.nist.gov/pml/data/micro/index.cfm and http://www. astro.uni-koeln.de/cdms/molecules
} 
complexity of the detected molecules is up to now limited to compounds with less than 14 atoms except for the recent detection of C60 and C70 in reflection nebulae (Sellgren et al. 2010; Cami et al. 2010). A huge amount of studies have been conducted to find the building blocks of life, and the detection of the simplest amino acid, glycine $\left(\mathrm{H}_{2} \mathrm{NCH}_{2} \mathrm{COOH}\right)$, has been erroneously reported several times (Snyder et al. 2005). Up to now, only one sugar, glycolaldehyde $\left(\mathrm{HOCH}_{2} \mathrm{CHO}\right)$, has been unambiguously identified (Hollis et al. 2000).

Some molecules are chiral, which means that they have two geometrical mirror-image forms that cannot be superimposed on each other. Chiral compounds play a particular role because life on Earth is to a significant part based on such compounds and on the homochirality of many of them, particularly the amino acids and sugars. Up to now, these chiral compounds, in abundance on Earth, have only been observed in meteorites generally after an aqueous acidic treatment. Unsuccessful attempts to discover a first chiral compound in the ISM have already been reported, and propylene epoxyde $\left(\mathrm{H}_{2} \mathrm{C}(\mathrm{O}) \mathrm{CHCH}_{3}\right)$ was the target (Jones et al. 2007; Cunningham et al. 2007). Many other chiral compounds could be present in the ISM with substituents largely represented in the list of detected molecules (Marloie et al. 2010; Lattelais et al. 2009). Some of them are potential precursors of these building blocks of life.

Even if it is not possible to distinguish between different enantiomers in astronomical radio spectra, the presence and the abundance of these chiral compounds in the interstellar medium is of interest for understanding the origin of the chiral species encountered in meteorites and for discussing models of the possible interstellar origin of the enantiomeric excess that have been reported in these relics of early solar system chemistry (Pizzarello \& Groy 2011; De Marcellus et al. 2010, and references therein). On the other hand, some of us have recently reported the microwave spectra of 3-amino-2-propenitrile $\left(\mathrm{H}_{2} \mathrm{NCH}=\mathrm{CHCN}\right)$ (Askeland et al. 2006) and 3-mercapto-2propenenitrile $(\mathrm{HSCH}=\mathrm{CHCN})(\mathrm{Cole}$ et al. 2007). These two compounds are prochiral, which means that they can be converted into new and different chiral molecules in a single chemical step. And these compounds, which are adducts of ammonia or hydrogen sulfide $\left(\mathrm{H}_{2} \mathrm{~S}\right)$ with cyanoacetylene $(\mathrm{H}-\mathrm{C} \equiv \mathrm{C}-\mathrm{CN})$, have been proposed as candidates for the ISM. We also tried to find a plausible reaction pathway for the formation in the ISM of the latter and of cyanoacetaldehyde $\left(\mathrm{NCCH}_{2} \mathrm{CHO}\right)$ (formally the rearranged product of the addition of water to $\mathrm{HC}_{3} \mathrm{~N}$ ) (Horn et al. 2008).

We report here the synthesis and the first study of the rotational spectrum of 2-aminopropionitrile (Fig. 1), and a search for this compound in the hot dense core Sagittarius B2(N), hereafter Sgr B2(N) for short, one of the currently best sources to search for new molecules in the interstellar medium. This chiral species has been selected on the basis of the recent interstellar detection of aminoacetonitrile in the ISM (Belloche et al. 2008a). In the laboratory, glycine can be formed by acidic aqueous treatment of aminoacetonitrile and alanine $\left(\mathrm{CH}_{3} \mathrm{CH}\left(\mathrm{NH}_{2}\right) \mathrm{COOH}\right)$, the simplest chiral, natural amino acid, by hydrolysis of 2-aminopropionitrile (Béjaud et al. 1975). The physical and chemical properties of 2-aminopropionitrile are still poorly known. This compound is stable a few days at room temperature in the liquid phase. In the gas phase, a thermolysis study (Guillemin et al. 1988) has shown that it decomposes into $\mathrm{HCN}$ and $\mathrm{CH}_{3} \mathrm{CH}=\mathrm{NH}$ at $800{ }^{\circ} \mathrm{C}$. All molecules named in this article, except 2-aminopropionitrile which is in (Fig. 1), are drawn in Fig. A.1.

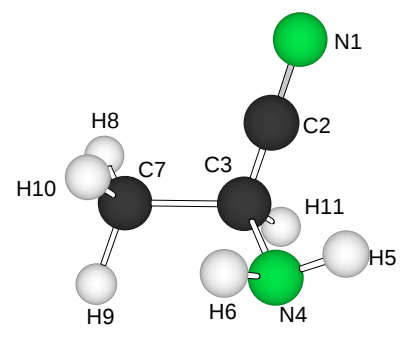

I

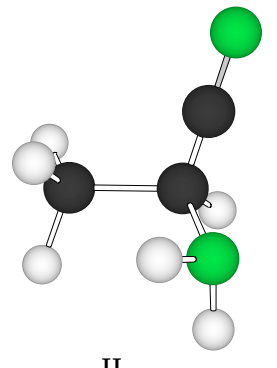

II

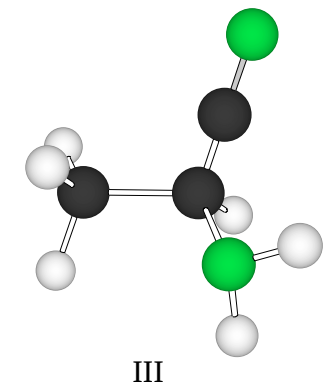

III
Fig. 1. Three stable conformers of 2-aminopropionitrile. Conformer I was calculated to be $8.0 \mathrm{~kJ} / \mathrm{mol}$ more stable than II and $6.8 \mathrm{~kJ} / \mathrm{mol}$ more stable than III employing the CCSD/cc-pVTZ procedure. The detailed CCSD structure is listed in the supporting information. Atom numbering is indicated on conformer $\mathrm{I}$, which is the preferred form of this compound.

\section{Experiments}

\subsection{Synthesis of 2-aminopropionitrile}

The laboratory synthesis of 2-aminopropionitrile has already been reported (Guillemin et al. 1988). The scheme of the reaction is represented in Fig. A.2.

\subsection{Oslo - Stark centimeter wave spectrometer}

The microwave spectrum of 2-aminopropionitrile was studied in the 22-80 GHz frequency interval by Stark-modulation spectroscopy using the microwave spectrometer of the University of Oslo. Details of the construction and operation of this device have been given elsewhere (Møllendal et al. 2005, 2006). This spectrometer has a resolution of about $0.5 \mathrm{MHz}$ and measures the frequency of isolated transitions with an estimated accuracy of $\approx 0.10 \mathrm{MHz}$. The experiments were performed at about $-30^{\circ} \mathrm{C}$ by cooling the $2 \mathrm{~m}$ Hewlett-Packard absorption cell with portions of dry ice. The cooling was performed to enhance the intensity of the spectrum.

\subsection{Lille-submillimeter wave spectrometer}

The submillimeter-wave measurements (150-660 GHz) were performed using the Lille spectrometer (Motiyenko et al. 2010). Solid-state sources were used in the frequency ranges 150-322 and 400-533 GHz. The frequency of the Agilent synthesizer $(12.5-17.5 \mathrm{GHz})$ was first multiplied by six and amplified by a Spacek active sextupler providing the output power of $+15 \mathrm{dBm}$ in the W-band range $(75-110 \mathrm{GHz})$. This power is high enough to use passive Schottky multipliers (X2, X3, X5) from Virginia Diodes Inc in the next stage of frequency multiplication chain. In the range from 580 to $660 \mathrm{GHz}$, the Istok backward wave oscillator (BWO) was used as a source of radiation. It is phase-locked to a harmonic of the Agilent E8257D synthesizer $(2-20 \mathrm{GHz})$. As a detector we used an InSb liquid He-cooled bolometer from 
QMC Instruments Ltd to improve the sensitivity of the spectrometer, the sources were frequency modulated at $10 \mathrm{kHz}$. The absorption cell is a stainless-steel tube $(6 \mathrm{~cm}$ diameter, $220 \mathrm{~cm}$ long). The sample pressure during measurements was about $2.5 \mathrm{~Pa}(25 \mu \mathrm{bar})$ and the linewidth was limited by Doppler broadening. These measurements were performed at room temperature. The measurement accuracy for isolated lines is estimated to be better than $30 \mathrm{kHz}$. However, if the lines were blended or had a poor signal-to-noise ratio, they were given a weight of 100 or even $200 \mathrm{kHz}$.

\section{Quantum chemical calculations}

\subsection{Methods}

The present ab initio and density functional theory (DFT) calculations were performed employing the Gaussian 03 suite of programs (Frisch et al. 2003) running on the Titan cluster in Oslo. Calculations were performed using three different methodologies, namely couple-cluster with single and double excitations (CCSD) (Purvis \& Bartlett 1982), Møller-Plesset secondorder perturbation calculations (MP2) (Møller \& Plesset 1934), and density functional theory (DFT) calculations employing Becke's three-parameter hybrid functional (Becke 1988) and the Lee, Yang and Parr correlation functional (B3LYP) (Lee et al. 1988). The Peterson and Dunnings (Peterson \& Dunning 2002) correlation-consistent triple- $\zeta$ wave function cc-pVTZ was employed in the CCSD calculations, and this function augmented with diffuse functions, aug-cc-pVTZ, was used in the MP2 calculations. The 6-311++G(3df, 3pd) wave function, which is also of triple- $\zeta$ quality and augmented with diffuse functions, was employed in the B3LYP calculations. The default convergence criteria of Gaussian 03 were observed in all computations.

\subsection{Results}

Rotation about the $\mathrm{C}-\mathrm{NH}_{2}$ bond that connects the amino group with the rest of the molecule may produce rotational isomerism. The quantum chemical calculations predict that three rotameric forms (conformers) exist for this compound. These conformers, denoted I, II, and III, are depicted in Fig. 1.

The structures, dipole moments, and energy differences were calculated for these forms at the very high CCSD/cc-pVTZ level of theory and the results are assumed to be accurate to within $3 \mathrm{~kJ} / \mathrm{mol}$. The full CCSD structures, dipole moment components, and energy differences, not corrected for zero-point vibrational effects, are given in Tables B.1 and B.2.

Interestingly, conformer $\mathrm{I}$ is predicted to be $7-8 \mathrm{~kJ} / \mathrm{mol}$ (840-960 K) more stable than II or III in the CCSD calculations, so the two last conformers need not be considered further because their populations are presumably very insignificant under the conditions prevailing in the ISM. Not surprisingly, conformer I uses both hydrogen atoms of the amino group to form intramolecular hydrogen bonds with the $\pi$-electrons of the cyano group, just as in the case of its congener aminoacetonitrile $\left(\mathrm{H}_{2} \mathrm{NCH}_{2} \mathrm{CN}\right.$ ) (Macdonald \& Tyler 1972; Pickett 1973), which has already been detected in the ISM (Belloche et al. 2008a).

Several additional calculations were performed, which include the potential function for rotation about the $\mathrm{C}-\mathrm{NH}_{2}$ bond (Fig. A.3), which was calculated at the B3LYP/6-311++G(3df, 3pd) level, MP2/aug-cc-pVTZ calculations of the harmonic and anharmonic vibrational frequencies reported in Table B.3, Watson's quartic centrifugal distortion constants (Watson 1977) and the vibration-rotation interaction constants (the $\alpha^{\prime}$ s) (Gordy \& Cook 1984) reported in Table B.4.

\section{Analysis of the rotational spectrum}

\subsection{The microwave region}

The CCSD/cc-pVTZ principal inertial axes dipole moment components of I were calculated to be $\mu_{a}=2.92, \mu_{b}=0.22$, and $\mu_{c}=0.37 \mathrm{D}$ (see Table B.2), which indicates that this form should have a comparatively strong a-type spectrum and very weak b- or c-type spectra. The spectrum of 2-aminopropionitrile was first observed in the microwave region and found to be comparatively simple, in accordance with these predictions. The assignment of a-type R-branch lines were readily made using the spectroscopic constants shown in Table 2 to predict their approximate frequencies.

The ground-state lines were accompanied by lines presumably belonging to vibrationally excited states: the anharmonic frequencies of the lowest vibrations are $184.7 \mathrm{~cm}^{-1}(265.8 \mathrm{~K})$ for the lowest skeletal bending vibration $\left(v_{27}\right), 225.6 \mathrm{~cm}^{-1}(324.6 \mathrm{~K})$ for the methyl torsion $\left(v_{26}\right), 238.2 \mathrm{~cm}^{-1}(342.7 \mathrm{~K})$ for the amino group torsion $\left(v_{25}\right)$, and $282.7 \mathrm{~cm}^{-1}(406.8 \mathrm{~K})$ for a skeletal bending mode $\left(v_{24}\right)$, according to the B3LYP calculations. Spectra of the first excited states of these fundamental vibrations were assigned. The spectrum of the second excited state of the lowest skeletal vibration $\left(v_{27}=2\right)$ was also assigned. The spectroscopic constants of these states are collected in Table 11. No splittings caused by torsion of the methyl group were observed for the ground and the first excited state of the methyl torsion $\left(v_{26}=1\right)$. The reason for this is that the coupling between the methyl group rotation, of the amino group rotation, and the overall rotation of the molecule, which might result in a resolved splitting of the microwave transitions, is comparatively small.

The assignments of these states were greatly facilitated by comparing the experimental vibration-rotation constants with their theoretical counterparts listed in Table B.4. Searches for the microwave spectra of the high-energy conformers II and III were performed using the CCSD spectroscopic constants (Table B.2) to predict the approximate frequencies of these spectra, but they were not identified, presumably because they were too weak owing to the very large energy differences $(8.0$ and $6.8 \mathrm{~kJ} / \mathrm{mol})$ between these hypothetical conformers and the preferred conformer I.

\subsection{The submillimeterwave region}

Thanks to the centimeterwave, the analysis of the spectra in the millimeter and submillimeterwave region was fairly easy to perform.

We note that some interaction between these lowest energy excited states (from 180 to $300 \mathrm{~cm}^{-1}$ ) occurs for high quantum numbers above $400 \mathrm{GHz}$. We had difficulties to obtain a very good fit. Nevertheless; it is possible to predict the excited states up to $400 \mathrm{GHz}$ very reliablely. The complete list of measurements is given in Table 1, available at the CDS, for the ground state and the excited vibrational states. Only part of the table is presented in this paper.

The final spectroscopic parameters obtained for the ground state and the excited states are provided in Tables 2 and B.5. Table 2 also lists the parameters calculated with the ab initio harmonic force field. The agreement is pretty good. This shows that the $\mathrm{ab}$ initio calculations can be useful for the assignment of new rotational spectra.

\subsection{Partition function}

When low-energy vibrationally excited states exist, the vibrational part of the partition function should be taken into account 
Table 1. Part of the table available in CDS, result of the fit for the ground state and the excited vibrational states.

\begin{tabular}{ccccccccccc}
\hline \hline$v_{\mathrm{t}}{ }^{\prime \prime}$ & $\begin{array}{c}J^{\prime \prime} \\
\text { Upper State }\end{array}$ & $K_{a}{ }^{\prime \prime}$ & $K_{c}{ }^{\prime \prime}$ & $v_{\mathrm{t}}{ }^{\prime}$ & $\begin{array}{c}J^{\prime} \\
\text { Lower State }\end{array}$ & $K_{a}{ }^{\prime}$ & $K_{c}{ }^{\prime}$ & $\begin{array}{c}\text { Obs. Freq. } \\
(\mathrm{MHz})\end{array}$ & $\begin{array}{c}\text { Obs-Calc } \\
(\mathrm{MHz})\end{array}$ & $\begin{array}{c}\text { Uncertainty } \\
(\mathrm{MHz})\end{array}$ \\
\hline 0 & 94 & 48 & 46 & 0 & 93 & 48 & 45 & 656835.850 & 0.002 & 0.050 \\
0 & 94 & 48 & 47 & 0 & 93 & 48 & 46 & 656835.850 & 0.002 & 0.050 \\
0 & 91 & 30 & 62 & 0 & 90 & 30 & 61 & 656987.505 & 0.068 & 0.050 \\
0 & 93 & 39 & 54 & 0 & 92 & 39 & 53 & 657023.535 & 0.028 & 0.050 \\
0 & 93 & 39 & 55 & 0 & 92 & 39 & 54 & 657023.535 & 0.028 & 0.050 \\
0 & 91 & 30 & 61 & 0 & 90 & 30 & 60 & 657227.864 & 0.058 & 0.030 \\
0 & 94 & 47 & 47 & 0 & 93 & 47 & 46 & 657517.887 & 0.043 & 0.030 \\
0 & 94 & 47 & 48 & 0 & 93 & 47 & 47 & 657517.887 & 0.043 & 0.030 \\
0 & 63 & 5 & 58 & 0 & 62 & 4 & 58 & 657715.704 & 0.032 & 0.030 \\
0 & 63 & 6 & 58 & 0 & 62 & 5 & 58 & 657715.704 & 0.032 & 0.030 \\
0 & 88 & 26 & 62 & 0 & 87 & 26 & 61 & 657814.623 & 0.047 & 0.030 \\
0 & 93 & 38 & 55 & 0 & 92 & 38 & 54 & 658132.920 & 0.037 & 0.050 \\
0 & 93 & 38 & 56 & 0 & 92 & 38 & 55 & 658132.920 & 0.037 & 0.050 \\
0 & 94 & 46 & 48 & 0 & 93 & 46 & 47 & 658229.007 & 0.039 & 0.050 \\
0 & 94 & 46 & 49 & 0 & 93 & 46 & 48 & 658229.007 & 0.039 & 0.050 \\
0 & 90 & 28 & 62 & 0 & 89 & 28 & 61 & 658562.443 & 0.058 & 0.050 \\
\hline
\end{tabular}

Table 2. Ground-state spectroscopic parameters of 2-aminopropionitrile.

\begin{tabular}{|c|c|c|c|}
\hline Parameters & Experimental $^{a}$ & $\mathrm{Ab}$ initio $^{b}$ & Diff in $\%$ \\
\hline$A$ (in $\mathrm{MHz}$ ) & $8329.21112(49)$ & 8396.94 & -0.81 \\
\hline$B($ in $\mathrm{MHz})$ & $3984.324764(53)$ & 3980.01 & 0.11 \\
\hline$C$ (in $\mathrm{MHz})$ & $2945.429514(51)$ & 2956.27 & -0.37 \\
\hline$\Delta_{J}($ in $\mathrm{kHz})$ & $1.002844(12)$ & 1.00 & 0.28 \\
\hline$\Delta_{J K}($ inkHz $)$ & $10.854560(80)$ & 10.7 & 1.42 \\
\hline$\Delta_{K}($ in $\mathrm{kHz})$ & $-3.6628(14)$ & -3.52 & 3.90 \\
\hline$\delta_{J}($ in $\mathrm{kHz})$ & $0.2375211(57)$ & 0.245 & -3.15 \\
\hline$\delta_{K}($ in $\mathrm{kHz})$ & $6.90720(13)$ & 6.77 & 1.99 \\
\hline$H_{J}($ in $\mathrm{Hz})$ & $0.0011965(10)$ & & \\
\hline$H_{J K}($ in $\mathrm{Hz})$ & $0.077216(27)$ & & \\
\hline$H_{K J}($ in $\mathrm{Hz})$ & $-0.126467(77)$ & & \\
\hline$H_{K}($ in $\mathrm{Hz})$ & $0.1560(13)$ & & \\
\hline$h_{J}($ in $\mathrm{Hz})$ & $0.00035469(52)$ & & \\
\hline$h_{J K}($ in $\mathrm{Hz})$ & $0.040765(32)$ & & \\
\hline$h_{K}($ in $\mathrm{Hz})$ & $0.19034(18)$ & & \\
\hline$L_{J J K}($ in $\mathrm{mHz})$ & $-0.00020315(99)$ & & \\
\hline$L_{K}($ in $\mathrm{mHz})$ & $-0.04097(17)$ & & \\
\hline$l_{J K}($ in $\mathrm{mHz})$ & $-0.0001107(19)$ & & \\
\hline \multicolumn{3}{|c|}{ Number of lines OSLO $(7-70 \mathrm{GHz})$} & 121 \\
\hline \multicolumn{3}{|c|}{ Standard deviation of OSLO data (in $\mathrm{kHz}$ ) } & 168 \\
\hline \multicolumn{3}{|c|}{ Number of lines LILLE (150-660 GHz) } & 1500 \\
\hline \multicolumn{3}{|c|}{ Standard deviation of LILLe data (in $\mathrm{kHz}$ ) } & 28 \\
\hline \multicolumn{3}{|l|}{$J_{\text {Max }}, K_{a, \text { Max }}$} & 99,56 \\
\hline \multicolumn{3}{|c|}{ Standard deviation of the fit (in $\mathrm{kHz}$ ) } & 54 \\
\hline \multicolumn{3}{|c|}{ Weighted deviation of fit } & 0.90 \\
\hline
\end{tabular}

Notes. ${ }^{(a)}$ Numbers in parenthesis are one time the standard deviation. (b) Harmonic force field calculated at the level MP2/aug-cc-pVTZ.

to derive column densities. Even at relatively low temperature $(150 \mathrm{~K})$ these states around $200 \mathrm{~cm}^{-1}$ are populated. The total partition function is given in Table 3. It was computed with the same approximation and equations as used by Widicus Weaver et al. (2005) for glycolaldehyde.

\section{Observations}

We observed the hot-core region Sgr B2(N) in January 2004, September 2004, and January 2005 with the IRAM $30 \mathrm{~m}$ telescope on Pico Veleta, Spain. We carried out a complete spectral survey in the $3 \mathrm{~mm}$ atmospheric window between 80
Table 3. Partition function of 2-aminopropionitrile.

\begin{tabular}{lcc}
\hline \hline$T$ in $\mathrm{K}$ & $Q_{\text {groundstate }}$ & $Q_{\text {total }}{ }^{a}$ \\
\hline 300 & 88750.34 & 221539.58 \\
225 & 57645.03 & 116321.72 \\
150 & 31377.98 & 46473.48 \\
75 & 11093.79 & 11731.97 \\
37.5 & 3922.25 & 3926.67 \\
18.75 & 1386.72 & 1386.73 \\
9.375 & 490.28 & 490.28 \\
\hline
\end{tabular}

Notes. ${ }^{(a)}$ The vibrational part includes the contribution of the five excited vibrational states $v_{27}=1, v_{26}=1, v_{25}=1, v_{24}=1$, and $v_{27}=2$.

and $116 \mathrm{GHz}$. A complete survey was performed in parallel in the $1.3 \mathrm{~mm}$ window between 201.8 and $204.6 \mathrm{GHz}$ and between 205.0 and $217.7 \mathrm{GHz}$. Additional selected spectra were also obtained in the $2 \mathrm{~mm}$ window and between 219 and $268 \mathrm{GHz}$. The angular resolution depends on the frequency $v$ and can be computed with the equation $H P B W\left({ }^{\prime \prime}\right)=2460 / v(\mathrm{GHz})$. The coordinates of the observed position are $\alpha_{\mathrm{J} 2000}=17^{\mathrm{h}} 47^{\mathrm{m}} 20.0$, $\delta_{\mathrm{J} 2000}=-28^{\circ} 22^{\prime} 19.0^{\prime \prime}$ with a systemic velocity $V_{\mathrm{lsr}}=64 \mathrm{~km} \mathrm{~s}^{-1}$. More details about the observational setup and the data reduction can be found in Belloche et al. (2008a). An rms noise level of $15-20 \mathrm{mK}$ on the $T_{\mathrm{a}}^{\star}$ scale was achieved below $100 \mathrm{GHz}$, 20-30 mK between 100 and $114.5 \mathrm{GHz}$, about $50 \mathrm{mK}$ between 114.5 and $116 \mathrm{GHz}$, and $25-60 \mathrm{mK}$ in the $2 \mathrm{~mm}$ window. At $1.3 \mathrm{~mm}$, the confusion limit was reached for most spectra.

\section{Column density upper limit toward Sgr B2(N)}

We searched for the transitions of 2-aminopropionitrile in our complete molecular line survey of Sgr B2(N) at $3 \mathrm{~mm}$ as well as in our partial surveys at 2 and $1.3 \mathrm{~mm}$. The search was conducted in a systematic way by modeling the expected emission with the XCLASS program ${ }^{2}$ in the local thermodynamic equilibrium (LTE), which is certainly a good approximation for the rotational transitions in the vibrational ground state given the very high

2 We made use of the XCLASS program (http://www.astro. uni-koeln.de/projects/schilke/XCLASS), which accesses the CDMS (Müller et al. 2001, 2005, http://www.cdms.de) and JPL (Pickett et al. 1998, http://spec.jpl.nasa.gov) molecular databases. 
Table 4. List of ten spectral features grouping transitions of 2-aminoproprionitrile observed toward Sgr B2(N).

\begin{tabular}{|c|c|c|c|c|c|c|c|c|c|c|c|c|c|}
\hline$F^{a}$ & $\mathrm{~S}^{b}$ & $N^{c}$ & $\begin{array}{l}\text { Transition } \\
\text { (4) }\end{array}$ & $\begin{array}{l}\text { Frequency } \\
(\mathrm{MHz}) \\
(5)\end{array}$ & $\begin{array}{c}\mathrm{Unc}^{d} \\
(\mathrm{kHz}) \\
(6)\end{array}$ & $\begin{array}{l}E_{1}^{e} \\
(\mathrm{~K}) \\
(7)\end{array}$ & $\begin{array}{l}S \mu^{2} \\
\left(\mathrm{D}^{2}\right) \\
(8)\end{array}$ & $\begin{array}{c}\sigma^{f} \\
(\mathrm{mK}) \\
(9)\end{array}$ & (10) & $\begin{array}{c}I_{\mathrm{obs}}{ }^{h} \\
\left(\mathrm{~K} \mathrm{~km} \mathrm{~s}^{-1}\right) \\
(11)\end{array}$ & $\begin{array}{l}I_{\mathrm{mod}}^{h} \\
(\mathrm{~K} \mathrm{kI} \\
(12)\end{array}$ & $\begin{array}{c}I_{\text {all }^{h}} \\
\left.\mathrm{n} \mathrm{s}^{-1}\right) \\
(13)\end{array}$ & $\begin{array}{c}\text { Comments } \\
\text { (14) }\end{array}$ \\
\hline F1 & $\mathrm{t}$ & 11 & $13_{3,11}-12_{3,10}$ & 88137.062 & 1 & 28 & 126.3 & 19 & 0.05 & $0.08(08)$ & 0.18 & 0.00 & Candidate detected line \\
\hline $\mathrm{F} 2$ & $\mathrm{c}$ & 17 & $15_{0,15}-14_{1,14}$ & 90722.899 & 2 & 31 & 0.5 & 14 & 0.13 & $-0.23(06)$ & 0.47 & -0.11 & $\begin{array}{l}\text { Group issue, blend with HNC in } \\
\text { absorption? }\end{array}$ \\
\hline - & - & 18 & $16_{10,7}-16_{9,7}$ & 90723.222 & 6 & 65 & 0.9 & - & - & - & - & - & - \\
\hline - & - & 19 & $16_{10,6}-16_{9,7}$ & 90723.250 & 6 & 65 & 0.2 & - & - & - & - & - & - \\
\hline - & - & 20 & $15_{1,15}-14_{1,14}$ & 90723.297 & 2 & 31 & 154.7 & - & - & - & - & - & - \\
\hline- & - & 21 & $15_{0,15}-14_{0,14}$ & 90723.819 & 2 & 31 & 154.7 & - & - & - & - & - & - \\
\hline - & - & 22 & $16_{10,7}-16_{9,8}$ & 90724.147 & 6 & 65 & 0.2 & - & - & - & - & - & - \\
\hline - & - & 23 & $16_{10,6}-16_{9,8}$ & 90724.176 & 6 & 65 & 0.9 & - & - & - & - & - & - \\
\hline - & - & 24 & $15_{1,15}-14_{0,14}$ & 90724.217 & 2 & 31 & 0.5 & - & - & - & - & - & - \\
\hline F3 & $\mathrm{t}$ & 56 & $14_{9,6}-13_{9,5}$ & 98328.023 & 1 & 49 & 86.1 & 18 & 0.07 & $0.16(07)$ & 0.28 & 0.01 & $\begin{array}{l}\text { Candidate detected group, } \\
\text { uncertain baseline }\end{array}$ \\
\hline- & - & 57 & $14_{9,5}-13_{9,4}$ & 98328.075 & 1 & 49 & 86.1 & - & - & - & - & - & - \\
\hline $\mathrm{F} 4$ & $\mathrm{t}$ & 72 & $16_{2,15}-15_{2,14}$ & 101362.165 & 2 & 39 & 161.0 & 16 & 0.07 & $0.16(06)$ & 0.31 & 0.09 & $\begin{array}{c}\text { Candidate detected line, uncertain } \\
\text { baseline }\end{array}$ \\
\hline F5 & $\mathrm{c}$ & 85 & $15_{11,5}-14_{11,4}$ & 105091.282 & 1 & 63 & 72.7 & 28 & 0.05 & $0.35(10)$ & 0.25 & 0.11 & $\begin{array}{l}\text { Group issue, blend with } \mathrm{U} \text {-line and } \\
\mathrm{C}_{2} \mathrm{H}_{3} \mathrm{CN}, v_{11}=2 \text {, uncertain baseline }\end{array}$ \\
\hline - & - & 86 & $15_{11,4}-14_{11,3}$ & 105091.282 & 1 & 63 & 72.7 & - & - & - & - & - & - \\
\hline F6 & $\mathrm{t}$ & 89 & $15_{9,7}-14_{9,6}$ & 105505.313 & 1 & 54 & 100.7 & 37 & 0.08 & $0.83(14)$ & 0.38 & 0.13 & Candidate detected line, noisy \\
\hline- & - & 90 & $15_{9,6}-14_{9,5}$ & 105505.510 & 1 & 54 & 100.7 & - & - & - & - & - & - \\
\hline F7 & $\mathrm{t}$ & 93 & $16_{3,14}-15_{3,13}$ & 106119.548 & 2 & 42 & 157.5 & 25 & 0.07 & $0.06(09)$ & 0.34 & 0.28 & Candidate detected line, noisy \\
\hline F8 & $\mathrm{c}$ & 106 & $36_{8,28}-36_{8,29}$ & 108387.475 & 5 & 241 & 24.2 & 48 & 0.17 & $2.53(19)$ & 0.81 & 4.13 & $\begin{array}{c}\text { Group issue?, blend with } \\
\mathrm{CH}_{3} \mathrm{C}(\mathrm{O}) \mathrm{CH}_{3},{ }^{13} \mathrm{CH}_{3} \mathrm{OH} \text {, and } \\
\mathrm{C}_{2} \mathrm{H}_{5} \mathrm{OH}\end{array}$ \\
\hline- & - & 107 & $18_{0,18}-17_{1,17}$ & 108389.721 & 2 & 45 & 0.6 & - & - & - & - & - & - \\
\hline- & - & 108 & $18_{1,18}-17_{1,17}$ & 108389.752 & 2 & 45 & 186.0 & - & - & - & - & - & - \\
\hline- & - & 109 & $18_{0,18}-17_{0,17}$ & 108389.794 & 2 & 45 & 186.0 & - & - & - & - & - & - \\
\hline- & - & 110 & $18_{1,18}-17_{0,17}$ & 108389.825 & 2 & 45 & 0.6 & - & - & - & - & - & - \\
\hline F9 & $\mathrm{t}$ & 119 & $17_{3,15}-16_{3,14}$ & 112029.767 & 2 & 47 & 167.8 & 29 & 0.08 & $0.89(09)$ & 0.39 & 0.02 & $\begin{array}{l}\text { Candidate detected line, partial } \\
\text { blend with U-line }\end{array}$ \\
\hline F10 & $\mathrm{c}$ & 126 & $16_{10,7}-15_{10,6}$ & 112426.272 & 1 & 63 & 102.3 & 31 & 0.08 & $0.81(11)$ & 0.42 & 0.48 & $\begin{array}{l}\text { Group issue?, noisy, partial blend } \\
\text { with t-HCOOH and } \mathrm{C}_{2} \mathrm{H}_{5} \mathrm{CN}\end{array}$ \\
\hline - & - & 127 & $16_{10,6}-15_{10,5}$ & 112426.294 & 1 & 63 & 102.3 & - & - & - & - & - & - \\
\hline
\end{tabular}

Notes. The ten spectral features group the transitions of 2-aminoproprionitrile that have been observed toward Sgr B2(N) with the IRAM $30 \mathrm{~m}$ telescope and are predicted to be strong enough $(>20 \mathrm{mK})$ and are not strongly blended with lines of other species according to our LTE modeling. (a) Numbering of the 2-aminopropionitrile features as in Fig. 2. The dashes indicate the transitions associated with the same feature. ${ }^{(b)}$ Status of each feature: "t" stands for tentative detection and "c" for possible conflict between the observed and modeled spectra. ${ }^{(c)}$ Numbering of the transitions associated with a modeled feature stronger than $20 \mathrm{mK}$ (see Table C.1). ${ }^{(d)}$ Frequency uncertainty. ${ }^{(e)}$ Lower energy level in temperature units $\left(E_{\mathrm{l}} / k_{\mathrm{B}}\right) .{ }^{(f)}$ Calculated rms noise level in $T_{\mathrm{mb}}$ scale. ${ }^{(g)}$ Peak opacity of the modeled feature. ${ }^{(h)}$ Integrated intensity in $T_{\mathrm{mb}}$ scale for the observed spectrum (Col. 11), the 2-aminopropionitrile model (Col. 12), and the model including all molecules detected so far except for 2-aminopropionitrile (Col. 13). The uncertainty in Col. 11 is given in parentheses in units of the last digit.

density characterizing the Sgr B2(N) hot core $\left(\sim 2 \times 10^{8} \mathrm{~cm}^{-3}\right.$, see, e.g., Belloche et al. 2008a,b). Because the partition function of 2-aminopropionitrile includes the contribution of five vibrationally and torsionally excited states also in the LTE approximation, we may overestimate its total column density if the density is not sufficient to thermally populate these vibrationally and torsionally excited states via collisions. However, infrared excitation may also contribute to populate the vibrationally excited states (e.g. Nummelin \& Bergman 1999), in which case we could underestimate the total column density. Finally, we cannot exclude that radiative decay involving ro-vibrational transitions may affect the rotational populations of the vibrational ground state. Since there is no easy way to correct for these possible non-LTE effects, we restrained our analysis to the LTE assumption. Because the spectrum of Sgr B2(N) is very close to the confusion limit, there are lines almost everywhere and the risk of misassignments is high. Therefore we used our complete model which includes all species detected in our survey so far (Belloche et al., in prep.; see Belloche et al. 2007, 2008a, 2009, for more details) to point out possible blends with transitions of other species.

Within the frequency ranges of our survey, 182 transitions of 2-aminopropionitrile are predicted to be stronger than $20 \mathrm{mK}$ on the main-beam temperature scale, which is a conservative limit given the typical noise level of this survey (see Sect. 5). They are listed in Table C.1, along with comments indicating whether they are blended with species included in our complete model of Sgr B2(N). Among these transitions, only 25 are found to be relatively free of contamination, and these are listed in Table 4. All other transitions of 2-aminopropionitrile are heavily blended with transitions of other species and cannot be detected with our single-dish data. A few of the 25 transitions are very close to each other in frequency so that they cannot be separated given the velocity dispersion that characterizes the molecular emission in Sgr B2(N). As a result, these 25 transitions are grouped into ten features, as listed in Col. 1 of Table 4. The LTE modeled spectrum of 2-aminopropionitrile is compared to the observed spectrum in Fig. 2, along with the complete model that includes all species identified in our survey so far. 

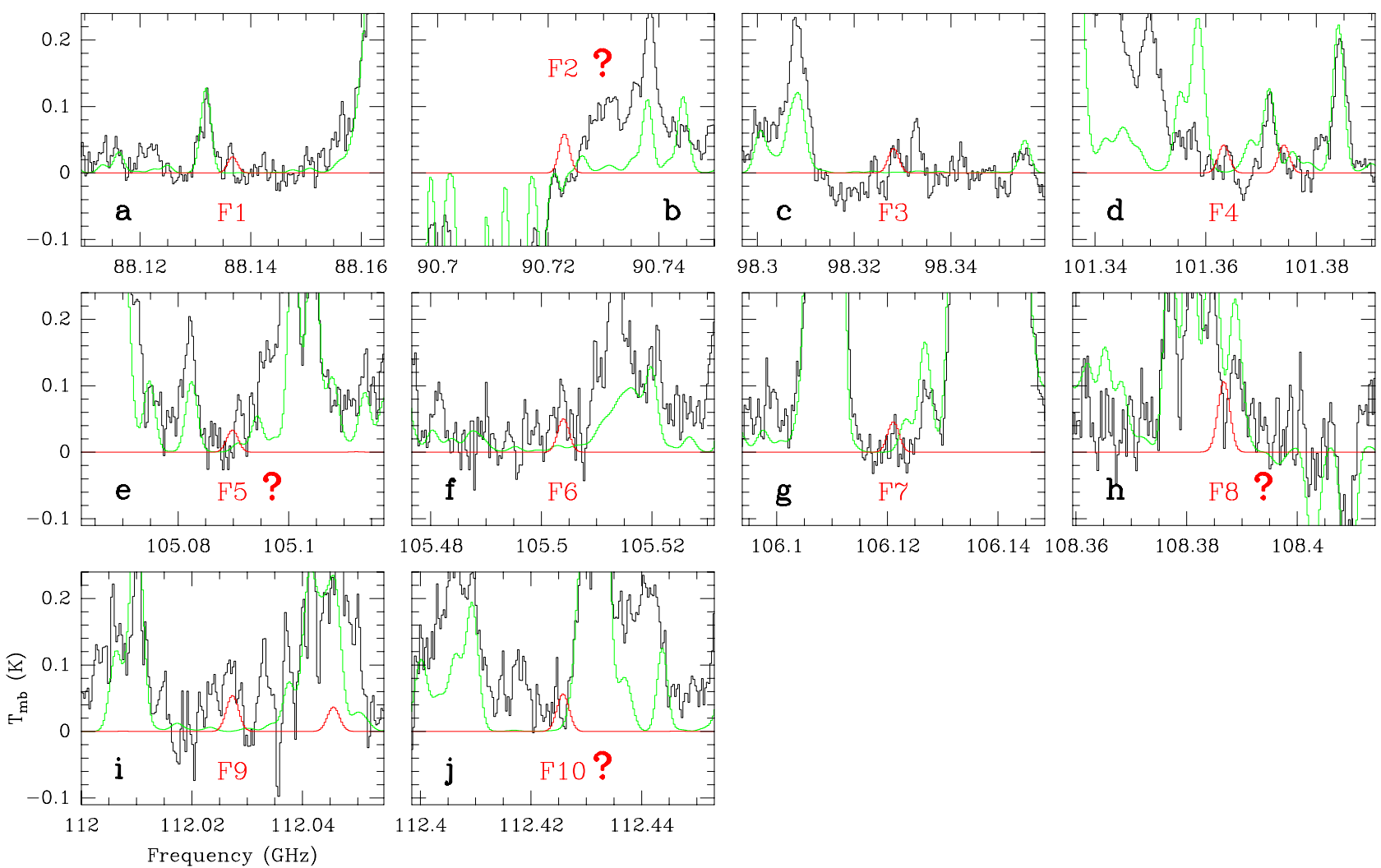

$\begin{array}{llllll}106.1 & 106.12 & 106.14 & 108.36 & 108.38 & 108.4\end{array}$

Fig. 2. Transitions of 2-aminopropionitrile tentatively detected toward Sgr B2(N) or possibly in conflict with the observed spectrum. The latter are indicated with a question mark. The spectrum taken with the IRAM $30 \mathrm{~m}$ telescope is shown in black on the main-beam temperature scale. The red spectrum is the LTE prediction for 2-aminopropionitrile based on the parameters listed in Table 5. The red labels are the feature numbers as in Col. 1 of Table 4. The green spectrum is the complete model that includes all molecules detected in the full line survey of Sgr B2(N) so far (except for 2-aminopropionitrile).

Table 5. Parameters of the LTE model of 2-aminopropionitrile.

\begin{tabular}{lcccc}
\hline \hline $\begin{array}{l}\text { Size }^{a} \\
(")\end{array}$ & $\begin{array}{r}T_{\text {rot }}{ }^{\prime} \\
(\mathrm{K})\end{array}$ & $\begin{array}{c}N^{c} \\
\left(\mathrm{~cm}^{-2}\right)\end{array}$ & $\begin{array}{c}\Delta V^{d} \\
\left(\mathrm{~km} \mathrm{~s}^{-1}\right)\end{array}$ & $\begin{array}{c}V_{\text {off }}{ }^{e} \\
\left(\mathrm{~km} \mathrm{~s}^{-1}\right)\end{array}$ \\
\hline 2.0 & $(2)$ & $(3)$ & $(4)$ & $(5)$ \\
\hline
\end{tabular}

Notes. This model is displayed in Fig. 2 and was used to derive the column density upper limit toward Sgr B2(N). ${ }^{(a)}$ Source diameter (FWHM). ${ }^{(b)}$ Temperature. ${ }^{(c)}$ Column density. ${ }^{(d)}$ Linewidth (FWHM). (e) Velocity offset with respect to the systemic velocity of Sgr B2(N) $V_{\mathrm{lsr}}=64 \mathrm{~km} \mathrm{~s}^{-1}$.

The physical parameters of the 2-aminopropionitrile model are given in Table 5. We assumed the same source size, temperature, linewidth, and systemic velocity as for aminoacetonitrile (Belloche et al. 2008a).

On the one hand, six of the ten features displayed in Fig. 2 are consistent with the observed spectrum and could be claimed as detected (F1, F3, F4, F6, F7, and F9), especially since the baseline level is uncertain and may have been overestimated in a few cases because of line confusion (F3, F4, and F7). However, four of these features have a low signal-to-noise ratio in terms of integrated intensity (F1, F3, F4, and F7, see Col. 11 of Table 4), and one (F9) may be blended with a still unidentified line. As a result, we consider these six features as tentatively detected only.

On the other hand, the four remaining features (F2, F5, F8, and F10) seem at first glance to be inconsistent with the observed spectrum. Feature F2 is blended with a redshifted velocity component of HNC 1-0 seen in absorption. Because the depth of this absorption is unknown, it could be stronger than assumed in our complete model and could significantly absorb the potential line of 2-aminopropionitrile. We checked for signs of a stronger absorption at the velocity corresponding to this component in the absorption spectra of other species $\left(\mathrm{HCN}, \mathrm{HCO}^{+}\right.$, $\mathrm{CS}, \mathrm{c}-\mathrm{C}_{3} \mathrm{H}_{2}$ ) but did not find any clear evidence that would confirm this option. The status of F2 is therefore unclear. Feature F5 does not show any sign of detection but because the modeled feature is weak and the emission-free channels in this portion of the observed spectrum are quasi inexistent, the baseline could have been slightly overestimated $(30 \mathrm{mK}$ would be sufficient to remove the inconsistency). Even if it is predicted to be a slightly stronger than F5, the case of feature F10 is very similar: the level of the baseline could have been overestimated in the observed spectrum, which would remove the small inconsistency. Therefore, the apparent non-detection of these two features does not rule out a possible detection of 2-aminopropionitrile. Finally, feature F8 peaks between two well detected lines that are found to be blends of $\mathrm{C}_{2} \mathrm{H}_{5}{ }^{13} \mathrm{CN}$ and $\left(\mathrm{CH}_{3}\right)_{2} \mathrm{CO}$ for the lower-frequency one and ${ }^{13} \mathrm{CH}_{3} \mathrm{OH}$ and $\mathrm{C}_{2} \mathrm{H}_{5} \mathrm{OH}$ for the higherfrequency one. The 2-aminopropionitrile feature does not have any observed counterpart at the position of its peak. The discrepancy in peak temperature is at the $\sim 2 \sigma$ level, but several channels show this inconsistency, so we believe it is significant. Because the baseline does not seem to have been overestimated in this part of the spectrum, F8 appears to be a more severe problem from than the three other features discussed above. As a result, we refrain claiming any tentative detection of aminopropionitrile and prefer to consider the LTE model of Table 5 as a sensitive $\sim 3 \sigma$ upper limit on the column density of this molecule. 


\section{Discussion}

In Sect. 6 we derived a column density upper limit of $1.7 \times$ $10^{16} \mathrm{~cm}^{-2}$ for 2-aminopropionitrile, assuming the source size, temperature, linewidth, and systemic velocity to be the same as for aminoacetonitrile. This number takes into account the contribution of the lowest energy vibrational-torsional modes ${ }^{3}$.

Belloche et al. (2009) reported an abundance ratio of 1.4 between methyl cyanide $\left(\mathrm{CH}_{3} \mathrm{CN}\right)$ and ethyl cyanide $\left(\mathrm{C}_{2} \mathrm{H}_{5} \mathrm{CN}\right)$ in $\mathrm{Sgr} \mathrm{B} 2(\mathrm{~N})$. Because aminoacetonitrile and 2-aminopropionitrile are based on the same carbon chains as these two alkyl cyanides, but with an additional amine functional group, one could naively expect the same abundance ratio. However, the amine group is at the end of the chain in the former molecule, while it is bound to the central carbon in the latter. If the chemical pathway leading to 2-aminopropionitrile in the interstellar medium is the photodissociation of an hydrogen atom from ethyl cyanide followed by the addition of an amine radical, then the statistical likelyhood to find the amine on the central carbon would be $40 \%$ only, provided the photodissociation energy is the same for all hydrogen atoms in the chain. Following the analogy with the pair methyl/ethyl cyanide, we could then expect an abundance ratio of aminoacetonitrile to 2-aminopropionitrile of $3.5(1.4 / 0.4)$, which would be consistent with the lower limit $(>1.8)$ derived above for this chiral molecule we assumed the same source size, temperature, linewidth, and systemic velocity as for aminoacetonitrile.

\section{Conclusion}

We measured (in the range $8-660 \mathrm{GHz}$ ) for the first time the rotational spectra of 2-aminopropionitrile. With the help of ab initio calculations, more than 4000 lines from the ground and five excited vibrationally and torsionally states were assigned. The assignment of these low-energy excited states is very important for calculating the partition function. Omitting the vibrationaltorsional part would underestimate the column density by $20 \%$ at $100 \mathrm{~K}$

2-aminopropionitrile was searched for in an unbiased, single-dish, spectral survey of the hot dense core Sgr B2(N). Six groups of transitions were tentatively detected in the $3 \mathrm{~mm}$ atmospheric window, but the LTE predictions for four other groups are not (or only marginally) consistent with the observed spectrum. As a result, only a column density upper limit of $1.7 \times 10^{16} \mathrm{~cm}^{-2}$ could be derived for this chiral molecule. It is at least 1.8 times less abundant than aminoacetonitrile. A simple comparison to the pair methyl/ethyl cyanide detected toward Sgr B2(N) suggests that 2-aminopropionitrile should be 3.5 times less abundant than aminoacetonitrile, which is consistent with the limit derived here.

Acknowledgements. We thank Rob Garrod for enlightening discussions about the interstellar chemistry. This work was supported by the Programme National "Physique et Chimie du Milieu Interstellaire". A.K. thanks The Research
Council of Norway for financial assistance through Contract 177540/V30. J.-C.G. thanks also the Centre National d'Études Spatiales (CNES) and the Program EPOV (INSU-CNRS) for financial support.

\section{References}

Askeland, E., Møllendal, H., Uggerud, E., et al. 2006, J. Phys. Chem. A, 110, 12572

Bailey, J., Chrysostomou, A., Hough, J. H., et al. 1998, Science, 281, 672

Becke, A. D. 1988, Phys. Rev. A, 38, 3098

Béjaud, M., Mion, L., Taillades, J., \& Commeyras, A. 1975, Tetrahedron, 31, 403

Belloche, A., Comito, C., Hieret, C., et al. 2007, in Molecules in Space and Laboratory, ed. J. L. Lemaire, \& F. Combes

Belloche, A., Menten, K. M., Comito, C., et al. 2008a, A\&A, 482, 179

Belloche, A., Menten, K. M., Comito, C., et al. 2008b, A\&A, 492, 769

Belloche, A., Garrod, R. T., Müller, H. S. P., et al. 2009, A\&A, 499, 215

Braakman, R., Blake, G. A., Belloche, A., \& Menten, K. M. 2010, ApJ, 724, 994

Cami, J., Bernard-Salas, J., Peeters, E., \& Malek, S. E. 2010, Science, Sept., 1180

Cole, G. C., Møllendal, H., Khater, B., \& Guillemin, J. C. 2007, J. Phys. Chem. A, 111,1259

Cunningham, M. R., Jones, P. A., Godfrey, P. D., et al. 2007, MNRAS, 376, 1201

De Marcellus, P., Meinert, C., Nuevo, M., et al. 2010, ApJ, 727, L27

Frisch, M. J., Trucks, G. W., Schlegel, H. B., et al. 2003, Gaussian 03, revision B.03, Gaussian, Inc., Pittsburgh PA

Gordy, W., \& Cook, R. L. 1984, Microwave Molecular Spectra, In Techniques of Chemistry (New York: John Wiley \& Sons), Vol. XVII

Guillemin, J. C., \& Denis, J. M. 1988, Tetrahedron, 44, 4431

Hollis, J. M., Lovas, F. J., \& Jewell, P. R. 2000, ApJ, 540, L107

Horn, A., Møllendal, H., \& Guillemin, J. C. 2008, J. Phys. Chem. A, 112, 11009 Joyce, G. F., Visser, G. M., Van Boeckel, C. A. A., et al. 1984, Nature, 310, 602

Jones, P. A., Cunningham, M. R., Godfrey, P. D., \& Cragg, D. M. 2007, MNRAS, 374, 579

Kuan, Y. J., Charnley, S. B., Huang, H. C., Tseng, W. L., \& Kisiel, Z. 2003, ApJ, 593,848

Lattelais, M., Pauzat, F., Ellinger, Y., \& Cecccarelli, C. 2009, ApJ, 696, L133

Lee, C., Yang, W., \& Parr, R. G. 1988, Phys. Rev. B, 37, 785

Macdonald, J. N., \& Tyler, J. K. 1972, J. Chem. Soc. Chem. Commun., 17, 995

Marloie, G., Lattelais, M., Pauzat, F., Pilme, J., \& Ellinger, Y. 2010, Interdisc. Sci. Comput. Life Sci., 2, 48

Møllendal, H., Leonov, A., \& de Meijere, A. 2005, J. Phys. Chem. A, 109, 6344 Møllendal, H., Cole, G. C., \& Guillemin, J. C. 2006, J. Phys. Chem. A, 110, 921 Møller, C., \& Plesset, M. S. 1934, Phys. Rev., 46, 618

Motiyenko, R. A., Margulès, L., Alekseev, E. A., Guillemin, J. C., \& Demaison, J. 2010, J. Mol. Spectrosc., 264, 94

Müller, H. S. P., Thorwirth, S., Roth, D. A., \& Winnewisser, G. 2001, A\&A, 370, L49

Müller, H. S. P., Schlöder, F., Stutzki, J., \& Winnewisser, G. 2005, J. Mol. Struct., 742,215

Nummelin, A., \& Bergman, P. 1999, A\&A, 341, L59

Peterson, K. A., \& Dunning, T. H., Jr. 2002, J. Chem. Phys., 117, 10548

Pickett, H. M. 1973, J. Mol. Spectrosc., 46, 335

Pickett, H. M., Poynter, I. R. L., Cohen, E. A., et al. 1998, J. Quant. Spectrosc. Radiat. Transfer, 60, 883

Pizzarello, S., \& Groy, T. L. 2011, Geochem. Cosmochim. Acta, 75, 645

Purvis, G. D., III, \& Bartlett, R. J. 1982, J. Chem. Phys., 76, 1910

Sellgren, K., Werner, M. W., Ingalls, J. G., et al. 2010, ApJ, 722, L54

Snyder, L. E., Lovas, F. J., Hollis, J. M., et al. 2005, ApJ, 619, 914

Watson, J. K. G. 1977, Vibrational Spectra and Structure (Amsterdam: Elsevier)

Widicus Weaver, S. L., Butler, R. A. H., Drouin, B. J., e al. 2005, ApJS, 158, 188

Pages 8 to 14 are available in the electronic edition of the journal at http://www. aanda. org

\footnotetext{
3 At $100 \mathrm{~K}$, the full partition function is 1.21 times larger than the partition function of the ground state. Because aminoacetonitrile was detected with a column density of $3.0 \times 10^{16} \mathrm{~cm}^{-2}$ (after taking into account the contribution of the lowest-energy vibrational modes to the partition function, see Belloche et al. 2008a; Braakman et al. 2010), 2-aminopropionitrile is at least 1.8 times less abundant than aminoacetonitrile in Sgr B2(N).
} 
Appendix A: Figures of the molecules discussed in the paper, the synthesis of 2-aminopropionitrile, and the ab initio potential curve<smiles>N#CCN</smiles>

Aminoacetonitrile<smiles>CC=O</smiles>

Acetaldehyde<smiles>CC#N</smiles>

Methyl cyanide

$$
\mathrm{H}-\mathrm{C} \equiv \mathrm{N}
$$

Hydrogen cyanide<smiles>O=CCO</smiles>

Glycolaldehyde<smiles>CCC#N</smiles>

Ethyl cyanide<smiles>NCC(=O)O</smiles>

Glycine<smiles>N#C/C=C\N</smiles><smiles>N#C/C=C\S</smiles><smiles>CC1CO1</smiles>

Methyl epoxide

Z-3-amino-2-propenenitrile

Z-3-mercapto-2-propenenitrile<smiles>S</smiles><smiles>N#CCC=O</smiles>

Cyanoacetaldehyde
$\mathrm{H}-\mathrm{C} \equiv \mathrm{C}-\mathrm{C} \equiv \mathrm{N}$

Cyanoacetylene<smiles>COC(C)C</smiles>

Alanine

Fig. A.1. Structure of the molecules discussed in the article. 


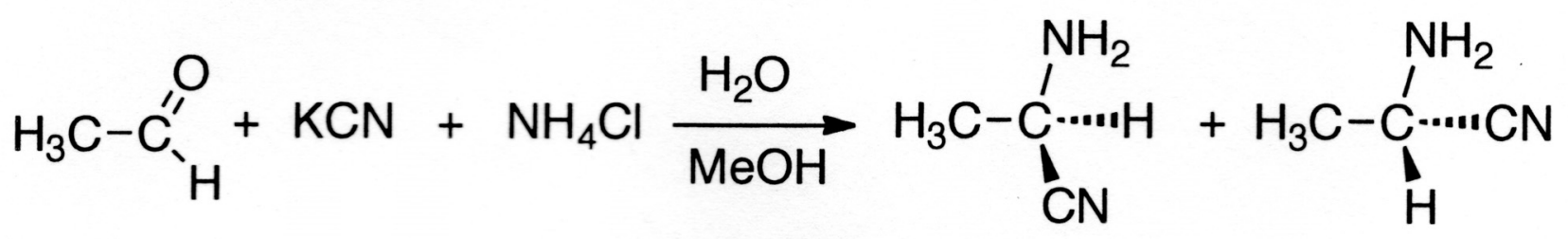

Fig. A.2. Scheme of the synthesis of 2-aminopropionitrile.

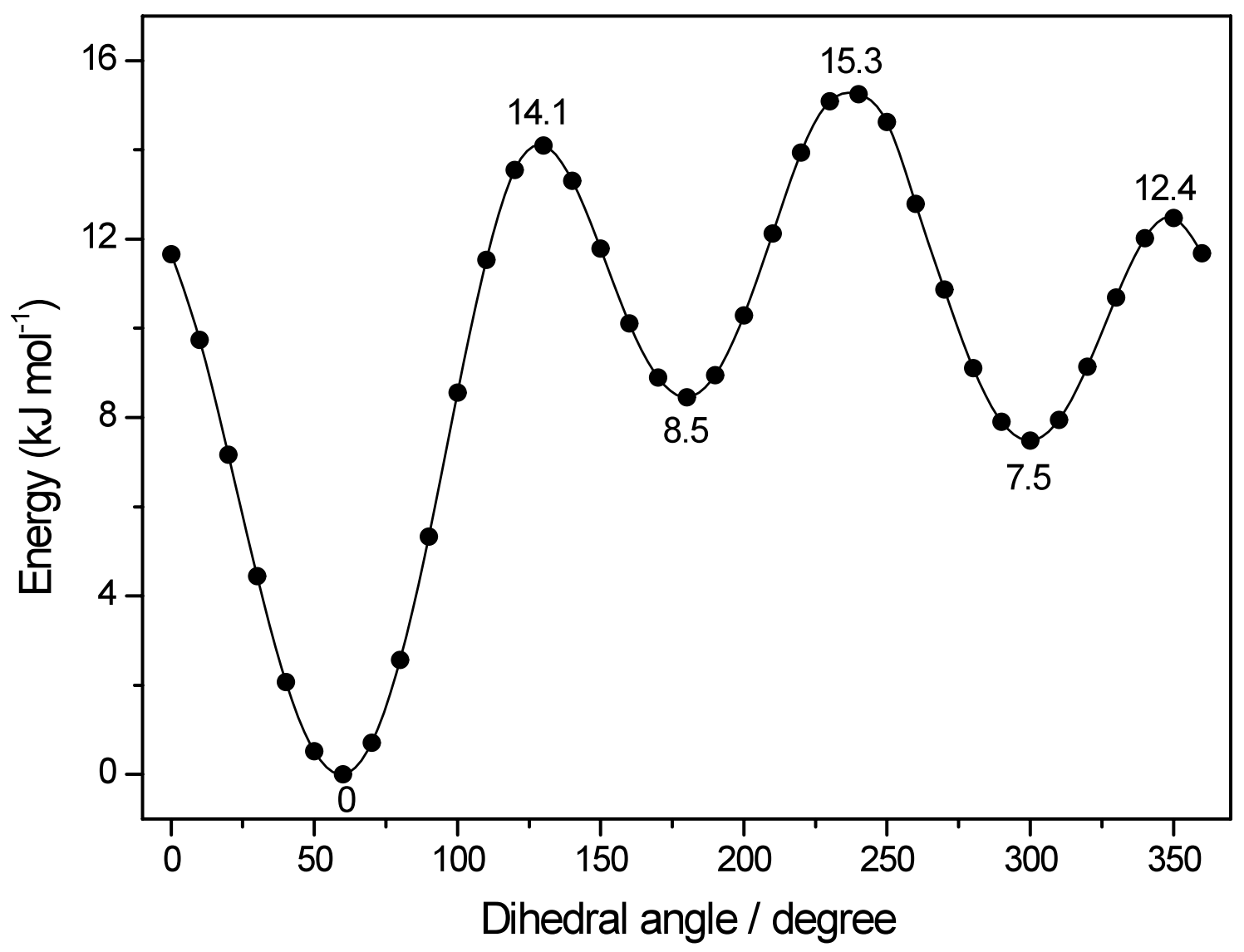

Fig. A.3. B3LYP/6-311++G(3df, 3pd) potential curve for the rotation about the C3-N4 bond. The abscissa is the H5-N4-C3-H11 dihedral angle. Conformer I has the lowest energy indicated by $0 \mathrm{~kJ} / \mathrm{mol}$ for dihedral angle of $59.4^{\circ}$. Rotamer II is higher in energy by 8.5 , and III by $7.5 \mathrm{~kJ} / \mathrm{mol}$ relative to I. The corresponding dihedral angles are 179.9 and $320^{\circ}$, respectively. The maxima have higher energies than I by $14.1,15.3$, and $12.4 \mathrm{~kJ} / \mathrm{mol}$, respectively. The corresponding dihedral angles are $128.8,236$, and $348.9^{\circ}$, respectively. 


\section{Appendix B: Tables of the results of ab initio calculations and spectroscopic parameters of the vibrationally excited states}

Table B.1. CCSD/cc-pVTZ Structures of three conformers of $\mathrm{CH}_{3}$ $\mathrm{CH}\left(\mathrm{NH}_{2}\right) \mathrm{CN}$.

\begin{tabular}{lrrr}
\hline \hline Conformer & \multicolumn{1}{c}{$\mathrm{I}^{a}$} & $\mathrm{II}$ & $\mathrm{III}$ \\
\hline & Bond rength & $\mathrm{pm})$ & \\
N1-C2 & 115.6 & 115.5 & 115.5 \\
C2-C3 & 148.6 & 147.8 & 147.8 \\
C3-N4 & 145.9 & 146.2 & 146.3 \\
C3-C7 & 152.5 & 153.2 & 152.6 \\
C3-H11 & 109.0 & 109.1 & 109.6 \\
N4-H5 & 101.2 & 101.3 & 101.2 \\
N4-H6 & 101.3 & 101.2 & 101.2 \\
C7-H8 & 108.9 & 109.0 & 108.9 \\
C7-H9 & 108.8 & 109.0 & 109.0 \\
C7-H10 & 109.1 & 109.0 & 108.8 \\
& & & \\
& & & \\
C2-C3-N4 & 113.1 & 108.5 & 108.5 \\
C2-C3-C7 & 110.0 & 109.6 & 110.2 \\
C2-C3-H11 & 106.8 & 106.5 & 106.0 \\
N4-C3-C7 & 110.0 & 115.5 & 109.6 \\
N4-C3-H11 & 107.7 & 107.3 & 113.5 \\
C7-C3-H11 & 109.1 & 109.2 & 108.9 \\
C3-N4-H5 & 110.3 & 109.6 & 108.6 \\
C3-N4-H6 & 109.5 & 108.8 & 110.3 \\
H5-N4-H6 & 106.4 & 106.6 & 106.7 \\
C3-C7-H8 & 111.0 & 111.3 & 110.9 \\
C3-C7-H9 & 109.0 & 109.4 & 109.4 \\
C3-C7-H10 & 110.3 & 110.3 & 109.8 \\
H8-C7-H9 & 109.6 & 108.7 & 108.5 \\
H8-C7-H10 & 108.3 & 108.5 & 109.3 \\
H9-C7-H10 & 108.8 & 108.6 & 108.8 \\
N1-C2-C3 & 178.0 & 177.8 & 179.0 \\
N1-C2-C7 & 144.0 & 141.9 & 144.6 \\
& & & \\
C2-C3-N4-H5 & -56.3 & 65.8 & -178.0 \\
C2-C3-N4-H6 & 60.5 & -178.1 & -61.3 \\
C7-C3-N4-H5 & -179.7 & -57.7 & 61.6 \\
C7-C3-N4-H6 & -63.0 & 58.4 & 178.2 \\
H11-C3-N4-H5 & 61.5 & -179.6 & -60.4 \\
H11-C3-N4-H6 & 178.2 & -63.5 & 56.3 \\
C2-C3-C7-H8 & 57.0 & 57.8 & 58.7 \\
C2-C3-C7-H9 & 177.7 & 177.9 & 178.4 \\
C2-C3-C7-H10 & -63.0 & -62.7 & -62.3 \\
N4-C3-C7-H8 & -177.8 & -179.4 & 178.1 \\
N4-C3-C7-H9 & -57.1 & -59.2 & -62.2 \\
N4-C3-C7-H10 & 62.2 & 60.2 & 57.1 \\
H11-C3-C7-H8 & -59.8 & -58.5 & -57.2 \\
H11-C3-C7-H9 & 60.9 & 61.7 & 62.5 \\
H11-C3-C7-H10 & -179.8 & -178.9 & -178.2 \\
\hline
\end{tabular}

Notes. ${ }^{(a)}$ The MW spectrum of this conformer was assigned.
Table B.2. CCSD/cc-pVTZ and MP2/aug-cc-pVTZ parameters of spectroscopic interest of three conformers ${ }^{a}$ of $\mathrm{CH}_{3} \mathrm{CH}\left(\mathrm{NH}_{2}\right) \mathrm{CN}$.

\begin{tabular}{|c|c|c|c|}
\hline Conformer & $I^{b}$ & II & III \\
\hline \multicolumn{4}{|c|}{ Rotational constants $(\mathrm{MHz})^{c}$} \\
\hline$A$ & 8381.0 & 8308.0 & 8533.8 \\
\hline$B$ & 3986.0 & 4025.8 & 4002.9 \\
\hline$C$ & 2955.1 & 2960.8 & 29510.2 \\
\hline \multicolumn{4}{|c|}{ Quartic centrifugal distortion constants $(\mathrm{kHz})$} \\
\hline$\Delta_{J}$ & 1.01 & 1.00 & 0.948 \\
\hline$\Delta_{J K}$ & 11.0 & 10.7 & 11.7 \\
\hline$\Delta_{K}$ & -3.28 & -3.52 & -3.68 \\
\hline$\delta_{J}$ & 0.233 & 0.245 & 0.235 \\
\hline$\delta_{K}$ & 6.97 & 6.77 & 7.42 \\
\hline \multicolumn{4}{|c|}{ Dipole moment $\left(10^{-30} \text { C.m }\right)^{c}$} \\
\hline$\mu_{a}$ & 9.73 & 16.24 & 14.60 \\
\hline$\mu_{b}$ & 0.75 & 3.37 & 0.90 \\
\hline$\mu_{c}$ & 1.24 & 0.49 & 6.62 \\
\hline \multicolumn{4}{|c|}{ Energy differences $(\mathrm{kJ} / \mathrm{mol})^{c, e}$} \\
\hline$\Delta E$ & 0.0 & 8.02 & 6.78 \\
\hline
\end{tabular}

Notes. ${ }^{(a)}$ Minima on the potential energy hypersurface. ${ }^{(b)}$ The rotational spectrum of this conformer was assigned. ${ }^{(c)}$ CCSD values. ${ }^{(d)}$ MP2 values. ${ }^{(e)}$ Electronic energy of I: $-595967.10 \mathrm{~kJ} / \mathrm{mol}$.

Table B.3. Harmonic and anharmonic vibrational fundamentals (in $\mathrm{cm}^{-1}$ ).

\begin{tabular}{lcc}
\hline \hline Mode & $E($ harm $)$ & $E($ anharm $)$ \\
\hline 1 & 3573.621 & 3406.863 \\
2 & 3494.839 & 3368.757 \\
3 & 3126.649 & 2984.423 \\
4 & 3110.063 & 2971.160 \\
5 & 3046.371 & 2904.660 \\
6 & 3040.428 & 2925.999 \\
7 & 2332.025 & 2301.749 \\
8 & 1662.248 & 1634.423 \\
9 & 1496.994 & 1478.080 \\
10 & 1491.705 & 1451.234 \\
11 & 1414.502 & 1383.232 \\
12 & 1389.675 & 1356.804 \\
13 & 1342.000 & 1302.193 \\
14 & 1256.291 & 1227.686 \\
15 & 1144.898 & 1111.567 \\
16 & 1088.629 & 1056.431 \\
17 & 1017.473 & 1000.901 \\
18 & 927.261 & 904.448 \\
19 & 864.262 & 774.854 \\
20 & 780.174 & 757.172 \\
21 & 583.180 & 578.410 \\
22 & 570.465 & 565.546 \\
23 & 390.256 & 389.433 \\
24 & 287.627 & 282.706 \\
25 & 250.003 & 238.255 \\
26 & 218.155 & 225.604 \\
27 & 189.231 & 184.783 \\
\hline & &
\end{tabular}


H. Møllendal et al.: Rotational spectrum of 2-aminopropionitrile and a search for it in Sagittarius B2(N)

Table B.4. B3LYP/6-311++G(3df, 3pd) vibration-rotation $\alpha$ matrix (MHz) of conformer I.

\begin{tabular}{|c|c|c|c|c|c|c|}
\hline Component & $a_{\text {calc }}$ & $a_{\mathrm{obs}}$ & $b_{\text {calc }}$ & $b_{\mathrm{obs}}$ & $c_{\text {calc }}$ & $c_{\mathrm{obs}}$ \\
\hline $\mathrm{Q}(1)$ & 4.41803 & & -0.10174 & & 0.12650 & \\
\hline Q( 2$)$ & 5.38448 & & 0.59795 & & 0.26257 & \\
\hline Q( 3$)$ & 4.46275 & & 0.10638 & & 0.55836 & \\
\hline $\mathrm{Q}(4)$ & 1.12677 & & 0.95356 & & 0.34951 & \\
\hline $\mathrm{Q}(5)$ & -1.07870 & & 1.79240 & & 1.00802 & \\
\hline$Q(6)$ & -1.94911 & & 1.74467 & & 0.68414 & \\
\hline $\mathrm{Q}(7)$ & 1.86154 & & 16.39344 & & 8.87901 & \\
\hline $\mathrm{Q}(8)$ & 9.08136 & & -0.71564 & & -0.18967 & \\
\hline Q( 9) & -8.89154 & & 0.47796 & & -4.16200 & \\
\hline $\mathrm{Q}(10)$ & -20.87936 & & -3.50122 & & -0.29885 & \\
\hline $\mathrm{Q}(11)$ & 33.84763 & & 3.61562 & & 4.77916 & \\
\hline $\mathrm{Q}(12)$ & 3.29407 & & 0.74391 & & -13.45593 & \\
\hline $\mathrm{Q}(13)$ & 16.25289 & & 0.36610 & & 11.89892 & \\
\hline $\mathrm{Q}(14)$ & 3.52735 & & -0.46125 & & 4.15893 & \\
\hline $\mathrm{Q}(15)$ & 22.73022 & & 3.08255 & & -2.02520 & \\
\hline$Q(16)$ & 13.75552 & & 4.20012 & & 7.17597 & \\
\hline $\mathrm{Q}(17)$ & 19.24469 & & 2.38232 & & 2.54073 & \\
\hline $\mathrm{Q}(18)$ & 31.55444 & & 1.68777 & & 5.38346 & \\
\hline $\mathrm{Q}(19)$ & 10.99376 & & 7.33048 & & 5.43973 & \\
\hline $\mathrm{Q}(20)$ & 5.63817 & & 11.42762 & & 7.44543 & \\
\hline $\mathrm{Q}(21)$ & -219.19986 & & 2.13092 & & -2.12429 & \\
\hline $\mathrm{Q}(22)$ & 208.96100 & & 2.87320 & & 4.47809 & \\
\hline $\mathrm{Q}(23)$ & 8.14465 & & -8.17647 & & 1.09370 & \\
\hline $\mathrm{Q}(24)$ & 19.00745 & 18.79 & -3.70255 & -3.44 & -1.94751 & -1.73 \\
\hline $\mathrm{Q}(25)$ & 2.98869 & 6.86 & -1.12084 & -1.45 & 0.77103 & 1.04 \\
\hline$Q(26)$ & -46.22269 & -51.48 & -2.14092 & -4.20 & -2.32025 & -4.05 \\
\hline $\mathrm{Q}(27=2)$ & 77.16004 & 81.58 & -24.13036 & -24.29 & 2.34778 & 2.01 \\
\hline $\mathrm{Q}(27)$ & 38.58002 & 41.32 & -12.06518 & -12.23 & 1.17389 & 0.99 \\
\hline
\end{tabular}

Table B.5. Spectroscopic parameters of the vibrationally excited states.

\begin{tabular}{|c|c|c|c|c|c|}
\hline Parameters & $v_{27=1}$ & $v_{26=1}$ & $v_{25=1}$ & $v_{24=1}$ & $v_{27=2}$ \\
\hline Energy $\left(\text { in } \mathrm{cm}^{-1}\right)^{a}$ & 184.7 & 225.6 & 238.2 & 282.7 & 370.8 \\
\hline$A($ in $\mathrm{MHz})$ & $8287.8917(20)$ & $8380.6862(41)$ & $8322.3521(34)$ & $8310.4161(31)$ & $8247.6331(42)$ \\
\hline$B$ (in $\mathrm{MHz}$ ) & $3996.55361(23)$ & $3988.52138(32)$ & $3985.77815(27)$ & $3987.76717(24)$ & $4008.61501(35)$ \\
\hline$C$ (in $\mathrm{MHz})$ & 2944.43923(13) & $2949.48281(25)$ & $2944.38523(23)$ & $2947.15900(16)$ & $2943.42120(27)$ \\
\hline$\Delta_{J}($ in $\mathrm{kHz})$ & $1.011705(75)$ & $1.026474(92)$ & $0.987738(82)$ & $0.993488(72)$ & $1.020469(95)$ \\
\hline$\Delta_{J K}($ in $\mathrm{kHz})$ & $10.23071(43)$ & $11.39062(74)$ & $10.78368(67)$ & $10.73352(53)$ & $9.61190(83)$ \\
\hline$\Delta_{K}($ in $\mathrm{kHz})$ & $-5.6202(79)$ & $-1.140(19)$ & $-3.594(15)$ & $-3.908(14)$ & $-7.125(20)$ \\
\hline$\delta_{J}($ in $\mathrm{kHz})$ & $0.248268(38)$ & $0.233476(52)$ & $0.235779(40)$ & $0.238122(35)$ & $0.259062(53)$ \\
\hline$\delta_{K}($ in $\mathrm{kHz})$ & $6.62698(75)$ & $7.1830(14)$ & $6.8368(13)$ & $6.7900(10)$ & $6.3394(13)$ \\
\hline$H_{J}($ in $\mathrm{Hz})$ & $0.001012(14)$ & $0.0^{b}$ & $0.0005169(72)$ & $0.0004899(55)$ & $0.0003095(90)$ \\
\hline$H_{J K}($ in $\mathrm{Hz})$ & $0.07024(39)$ & $0.05593(29)$ & $0.05454(26)$ & $0.07110(35)$ & $0.04602(73)$ \\
\hline$H_{K J}($ in $\mathrm{Hz})$ & $-0.15863(93)$ & $-0.00724(35)$ & $-0.02400(36)$ & $-0.09684(89)$ & $-0.1491(21)$ \\
\hline$H_{K}($ in $\mathrm{Hz})$ & $0.0^{b}$ & $0.0^{b}$ & $0.0^{b}$ & $0.0^{b}$ & $0.0^{b}$ \\
\hline$h_{J}($ in $\mathrm{Hz})$ & $0.0002927(77)$ & $-0.0002907(39)$ & $0.0^{b}$ & $0.0^{b}$ & $0.0^{b}$ \\
\hline$h_{J K}($ in $\mathrm{Hz})$ & $0.03793(12)$ & $0.01795(23)$ & $0.02145(23)$ & $0.03282(17)$ & $0.03283(28)$ \\
\hline$h_{K}($ in $\mathrm{Hz})$ & $0.1567(21)$ & $0.0^{b}$ & $0.0^{b}$ & $0.1421(16)$ & $0.0452(38)$ \\
\hline$L_{J J K}($ in $\mathrm{mHz})$ & $-0.000568(22)$ & & & & \\
\hline$l_{K J}($ in $\mathrm{mHz})$ & $-0.00399(18)$ & & & & \\
\hline Number of lines & 679 & 465 & 453 & 505 & 412 \\
\hline Frequency max (in GHz) & 605 & 465 & 465 & 465 & 465 \\
\hline$J_{\mathrm{Max}}, K_{a, \mathrm{Max}}$ & 99,35 & 78,30 & 78,35 & 78,35 & 78,31 \\
\hline Standard deviation of the fit (in $\mathrm{kHz}$ ) & 77 & 89 & 84 & 81 & 88 \\
\hline Weighted deviation of fit & 0.96 & 1.18 & 1.04 & 0.87 & 1.06 \\
\hline
\end{tabular}

Notes. ${ }^{(a)} \mathrm{Ab}$ initio values calculated from the anharmonic force field at the level B3LYP/6-311++G(3df, $\left.3 \mathrm{pd}\right) .{ }^{(b)}$ Fixed to this value. 
Table C.1. Transitions of 2-aminopropionitrile observed with the IRAM $30 \mathrm{~m}$ telescope toward Sgr B2(N).

\begin{tabular}{|c|c|c|c|c|c|c|c|}
\hline$N^{a}$ & $\begin{array}{c}\text { Transition }^{b} \\
\text { (2) }\end{array}$ & $\begin{array}{l}\text { Frequency } \\
\text { (MHz) } \\
\text { (3) }\end{array}$ & $\begin{array}{c}\text { Unc. }^{c} \\
(\mathrm{kHz}) \\
(4)\end{array}$ & $\begin{array}{l}E_{1}^{d} \\
(\mathrm{~K}) \\
(5)\end{array}$ & $\begin{array}{l}S \mu^{2} \\
\left(\mathrm{D}^{2}\right) \\
(6)\end{array}$ & $\begin{array}{c}\sigma^{e} \\
(\mathrm{mK}) \\
(7)\end{array}$ & $\begin{array}{c}\text { Comments }^{f} \\
\text { (8) }\end{array}$ \\
\hline 1 & $13_{2,12}-12_{2,11}$ & 83676.207 & 2 & 26 & 129.8 & 16 & Blend with U-line \\
\hline 2 & $12_{2,10}-11_{2,9}$ & 83682.092 & 2 & 24 & 117.0 & 16 & Strong $\mathrm{SO}_{2}$ \\
\hline 3 & $13_{1,12}-12_{1,11}$ & 83771.532 & 2 & 26 & 129.8 & 16 & Blend with $\mathrm{C}_{2} \mathrm{H}_{3} \mathrm{~N}, v_{11}=3, \mathrm{C}_{3} \mathrm{H}_{7} \mathrm{CN}$, and U-line \\
\hline 4 & $12_{8,5}-11_{8,4}$ & 84232.232 & 1 & 37 & 69.9 & 19 & Blend with $\mathrm{CH}_{3} \mathrm{OCHO}$ \\
\hline 5 & $12_{8,4}-11_{8,3}$ & 84232.354 & 1 & 37 & 69.9 & 19 & Blend with $\mathrm{CH}_{3} \mathrm{OCHO}$ \\
\hline 6 & $9_{3,6}-8_{2,6}$ & 84831.675 & 2 & 14 & 1.1 & 22 & Blend with U-line \\
\hline 7 & $14_{0,14}-13_{1,13}$ & 84833.097 & 2 & 27 & 0.5 & 22 & Blend with U-line \\
\hline 8 & $14_{1,14}-13_{1,13}$ & 84834.016 & 2 & 27 & 144.2 & 22 & Blend with U-line \\
\hline 9 & $14_{0,14}-13_{0,13}$ & 84835.204 & 2 & 27 & 144.2 & 22 & Blend with U-line \\
\hline 10 & $14_{1,14}-13_{0,13}^{0,13}$ & 84836.124 & 2 & 27 & 0.5 & 22 & Blend with U-line \\
\hline 11 & $13_{3,11}-12_{3,10}^{0,13}$ & 88137.062 & 1 & 28 & 126.3 & 19 & Candidate detected line \\
\hline 12 & $12_{3,9}-11_{3,8}$ & 88651.177 & 1 & 25 & 117.4 & 17 & Strong $\mathrm{HCN}$ in absorption \\
\hline 13 & $12,8,-11_{4,7}$ & 88659.222 & 2 & 26 & 112.5 & 17 & Strong HCN in absorption \\
\hline 14 & $13_{2,11}-12_{2,10}$ & 89261.038 & 2 & 28 & 126.9 & 16 & Blend with $\mathrm{CH}_{3}{ }^{13} \mathrm{CH}_{2} \mathrm{CN}$ and $\mathrm{C}_{2} \mathrm{H}_{5} \mathrm{CN}, v_{13}=1 / v_{21}=1$ \\
\hline 15 & $14_{2,13}-13_{2,12}$ & 89578.953 & 2 & 30 & 140.2 & 18 & Strong $\mathrm{C}_{2} \mathrm{H}_{5} \mathrm{CN}$ and $\mathrm{C}_{2} \mathrm{H}_{5} \mathrm{CN}, v_{13}=1 / v_{21}=1$ \\
\hline 16 & $14_{1,13}-13_{1,12}^{2,12}$ & 89626.635 & 2 & 30 & 140.2 & 18 & Strong $\mathrm{C}_{2} \mathrm{H}_{5} \mathrm{CN}$ \\
\hline 17 & $15_{0,15}^{1,14}-14_{1,14}$ & 90722.899 & 2 & 31 & 0.5 & 14 & Group issue, blend with HNC in absorption? \\
\hline 18 & $16_{10,7}-16_{9,7}^{\star}$ & 90723.222 & 6 & 65 & 0.9 & 14 & Group issue, blend with HNC in absorption? \\
\hline 20 & $15_{1,15}-14_{1,14}$ & 90723.297 & 2 & 31 & 154.7 & 14 & Group issue, blend with HNC in absorption? \\
\hline 21 & $15_{0,15}-14_{0,14}$ & 90723.819 & 2 & 31 & 154.7 & 14 & Group issue, blend with HNC in absorption? \\
\hline 22 & $16_{10,7}-16_{9,8}^{\star}$ & 90724.147 & 6 & 65 & 0.2 & 14 & Group issue, blend with HNC in absorption? \\
\hline 24 & $15_{1,15}-14_{0,14}$ & 90724.217 & 2 & 31 & 0.5 & 14 & Group issue, blend with HNC in absorption? \\
\hline 25 & $13_{9,5}-12_{9,4}{ }^{\star}$ & 91181.395 & 1 & 45 & 71.0 & 25 & Noisy \\
\hline 27 & $13_{4,10}-12_{4,9}$ & 91298.378 & 1 & 30 & 122.3 & 25 & Blend with $\mathrm{CH}_{3} \mathrm{NH}_{2}, \mathrm{HC}^{13} \mathrm{CCN}, v_{5}=1 / v_{7}=3$, and U-line \\
\hline 28 & $13_{8,6}-12_{8,5}$ & 91397.221 & 1 & 41 & 84.7 & 25 & Blend with U-lines \\
\hline 29 & $13_{8,5}^{0,0}-12_{8,4}$ & 91397.700 & 1 & 41 & 84.7 & 25 & Blend with U-lines \\
\hline 30 & $13_{6,8}-12_{6,7}$ & 92145.504 & 1 & 35 & 107.3 & 27 & Noisy, uncertain baseline \\
\hline 31 & $7_{5,3}-6_{4,2}$ & 92341.054 & 5 & 11 & 0.2 & 22 & Noisy \\
\hline 32 & $13_{6,7}-12_{6,6}$ & 92341.178 & 1 & 35 & 107.2 & 22 & Noisy \\
\hline 33 & $13_{5,9}-12_{5,8}$ & 92376.802 & 1 & 32 & 115.9 & 22 & Noisy \\
\hline 34 & $13_{5,8}-12_{5,7}$ & 94049.350 & 2 & 32 & 116.2 & 31 & Noisy, blend with $\mathrm{CH}_{3} \mathrm{NH}_{2}$ \\
\hline 35 & $14_{3,12}-13_{3,11}$ & 94194.640 & 2 & 32 & 136.7 & 31 & Noisy \\
\hline 36 & $14_{2,12}-13_{2,11}$ & 94878.534 & 2 & 32 & 137.0 & 28 & Blend with U-line \\
\hline 37 & $24_{3,21}-24_{3,22}$ & 94878.974 & 6 & 98 & 11.6 & 28 & Blend with U-line \\
\hline 38 & $24_{3,21}-24_{2,22}$ & 94879.607 & 6 & 98 & 0.3 & 28 & Blend with U-line \\
\hline 39 & $13_{3,10}-12_{3,9}$ & 95126.083 & 1 & 29 & 127.1 & 28 & Blend with $\mathrm{C}_{2} \mathrm{H}_{3} \mathrm{CN}, v_{15}=1$ \\
\hline 40 & $8_{6,3}-8_{4,4}$ & 95126.973 & 11 & 16 & 0.2 & 28 & Blend with $\mathrm{C}_{2} \mathrm{H}_{3} \mathrm{CN}, v_{15}=1$ \\
\hline 41 & $15_{2,14}-14_{2,13}$ & 95472.758 & 2 & 34 & 150.6 & 23 & Blend with U-line \\
\hline 42 & $15_{1,14}-14_{1,13}$ & 95496.037 & 2 & 34 & 150.6 & 23 & Blend with $\mathrm{C}_{2} \mathrm{H}_{3} \mathrm{CN}, v_{11}=2$ \\
\hline 43 & $16_{0,16}-15_{1,15}$ & 96612.144 & 2 & 36 & 0.6 & 29 & Blend with $\mathrm{CH}_{3} \mathrm{OCHO}$ \\
\hline 44 & $16_{1,16}-15_{1,15}$ & 96612.315 & 2 & 36 & 165.1 & 29 & Blend with $\mathrm{CH}_{3} \mathrm{OCHO}$ \\
\hline 45 & $16_{0,16}-15_{0,15}$ & 96612.542 & 2 & 36 & 165.1 & 29 & Blend with $\mathrm{CH}_{3} \mathrm{OCHO}$ \\
\hline 46 & $16_{1,16}-15_{0,15}^{5}$ & 96612.713 & 2 & 36 & 0.6 & 29 & Blend with $\mathrm{CH}_{3} \mathrm{OCHO}$ \\
\hline 47 & $13_{4,9}-124,8$ & 96648.400 & 2 & 31 & 124.4 & 29 & Blend with $\mathrm{CH}_{3} \mathrm{C}(\mathrm{O}) \mathrm{CH}_{3}$ and $\mathrm{CH}_{3} \mathrm{OCHO}$ \\
\hline 48 & $37_{17,21}-36_{18,19}$ * & 97854.555 & 6 & 299 & 0.5 & 20 & Blend with $\mathrm{C}_{2} \mathrm{H}_{5} \mathrm{OH}$ and U-line \\
\hline 50 & $14_{4,11}-13_{4,10}$ & 97854.686 & 1 & 34 & 133.1 & 20 & Blend with $\mathrm{C}_{2} \mathrm{H}_{5} \mathrm{OH}$ and U-line \\
\hline 51 & $14_{11,3}-13_{11,2}^{\star}$ & 97991.385 & 1 & 59 & 56.2 & 20 & Strong $\mathrm{CS}$ and $\mathrm{CH}_{3} \mathrm{OCH}_{3}$ \\
\hline 53 & $51_{11,40}-50_{13,37}$ & 98133.740 & 10 & 481 & 1.6 & 20 & Blend with $\mathrm{CH}_{3}{ }^{13} \mathrm{CH}_{2} \mathrm{CN}$ \\
\hline 54 & $14_{10,5}-13_{10,4}^{\star}$ & 98135.123 & 1 & 54 & 71.9 & 20 & Blend with $\mathrm{CH}_{3}{ }^{13} \mathrm{CH}_{2} \mathrm{CN}$ \\
\hline 56 & $14_{9,6}-13_{9,5}^{\star}$ & 98328.023 & 1 & 49 & 86.1 & 18 & Candidate detected group, uncertain baseline \\
\hline 58 & $14_{8,7}-13_{8,6}$ & 98598.677 & 1 & 45 & 98.9 & 18 & Strong $\mathrm{C}_{2} \mathrm{H}_{5} \mathrm{CN}, v_{13}=1 / v_{21}=1, \mathrm{CH}_{3} \mathrm{C}(\mathrm{O}) \mathrm{CH}_{3}$, and $\mathrm{C}_{2} \mathrm{H}_{5} \mathrm{CN}, v_{20}=1$ \\
\hline 59 & $14_{8,6}-13_{8,5}$ & 98600.317 & 1 & 45 & 98.9 & 18 & Strong $\mathrm{C}_{2} \mathrm{H}_{5} \mathrm{CN}, v_{13}=1 / v_{21}=1, \mathrm{CH}_{3} \mathrm{C}(\mathrm{O}) \mathrm{CH}_{3}$, and $\mathrm{C}_{2} \mathrm{H}_{5} \mathrm{CN}, v_{20}=1$ \\
\hline 60 & $14_{7,8}-13_{7,7}$ & 98990.437 & 1 & 42 & 110.1 & 18 & Blend with U-line \\
\hline 61 & $14_{7,7}-13_{7,6}$ & 99024.247 & 1 & 42 & 110.1 & 19 & Strong $\mathrm{H}_{\alpha}$ recombination line \\
\hline 62 & $14_{6,9}-13_{6,8}$ & 99474.544 & 1 & 39 & 119.8 & 19 & Blend with $\mathrm{CH}_{3} \mathrm{C}(\mathrm{O}) \mathrm{CH}_{3}, v_{\mathrm{t}}=1, \mathrm{CH}_{3} \mathrm{C}^{13} \mathrm{CH}$, and U-line \\
\hline 63 & $14_{5,10}-13_{5,9}$ & 99501.397 & 1 & 37 & 127.7 & 19 & Blend with $\mathrm{C}_{2} \mathrm{H}_{5} \mathrm{CN}$ \\
\hline 64 & $14_{6,8}-13_{6,7}$ & 99906.338 & 1 & 39 & 119.8 & 14 & Blend with $\mathrm{HCC}^{13} \mathrm{CN}, v_{7}=1$ \\
\hline 65 & $15_{3,13}-14_{3,12}$ & 100180.686 & 2 & 37 & 147.1 & 14 & Blend with U-line \\
\hline 66 & $18_{11,8}-18_{10,8^{\star}}$ & 100182.883 & 6 & 81 & 1.0 & 14 & Blend with U-line \\
\hline 68 & $18_{11,8}-18_{10,9^{\star}}$ & 100183.254 & 6 & 81 & 0.3 & 14 & Blend with U-line \\
\hline 70 & $15_{2,13}-14_{2,12}$ & 100572.114 & 2 & 37 & 147.2 & 20 & $\begin{array}{l}\text { Blend with } \mathrm{NH}_{2} \mathrm{CHO}, \mathrm{HC}^{13} \mathrm{CCN}, v_{5}=1 / v_{7}=3 \text {, and } \\
\qquad \mathrm{CH}_{3} \mathrm{C}(\mathrm{O}) \mathrm{CH}_{3}, v_{\mathrm{t}}=1\end{array}$ \\
\hline 71 & $14_{3,11}-13_{3,10}$ & 101132.384 & 2 & 34 & 136.4 & 21 & Blend with $\mathrm{CH}_{3} \mathrm{C}(\mathrm{O}) \mathrm{CH}_{3}, v_{\mathrm{t}}=1, \mathrm{C}_{2} \mathrm{H}_{5} \mathrm{OH}$, and U-line \\
\hline
\end{tabular}


Table C.1. continued.

\begin{tabular}{|c|c|c|c|c|c|c|c|}
\hline$(1)$ & Transition $^{b}$ & $\begin{array}{c}\text { Frequency } \\
(\mathrm{MHz}) \\
(3)\end{array}$ & $\begin{array}{c}\text { Unc. }^{c} \\
(\mathrm{kHz}) \\
(4)\end{array}$ & $\begin{array}{c}E_{1}^{d} \\
(\mathrm{~K}) \\
(5) \\
\end{array}$ & $\begin{array}{l}S \mu^{2} \\
\left(\mathrm{D}^{2}\right) \\
(6) \\
\end{array}$ & $\begin{array}{c}\sigma^{e} \\
(\mathrm{mK}) \\
(7)\end{array}$ & $\begin{array}{c}\text { Comments }^{f} \\
(8)\end{array}$ \\
\hline 72 & $16_{2,15}-15_{2,14}$ & 101362.165 & 2 & 39 & 161.0 & 16 & Candidate detected line, uncertain baseline \\
\hline 73 & $16_{1,15}-15_{1,14}$ & 101373.308 & 2 & 39 & 161.0 & 16 & $\begin{array}{c}\text { Blend with } \mathrm{CH}_{3} \mathrm{OCHO} \text { and } \mathrm{C}_{2} \mathrm{H}_{5} \mathrm{CN}, v_{13}=1 / v_{21}=1 \text {, uncertain } \\
\text { baseline }\end{array}$ \\
\hline 74 & $48_{14,35}-47_{16,32}$ & 101374.224 & 8 & 443 & 2.0 & 16 & $\begin{array}{c}\text { Blend with } \mathrm{CH}_{3} \mathrm{OCHO} \text { and } \mathrm{C}_{2} \mathrm{H}_{5} \mathrm{CN}, v_{13}=1 / v_{21}=1 \text {, uncertain } \\
\text { baseline }\end{array}$ \\
\hline 75 & $14_{5,9}-13_{5,8}$ & 102276.096 & 2 & 37 & 128.3 & 30 & Noisy, blend with $\mathrm{C}_{2} \mathrm{H}_{5} \mathrm{OH}$ \\
\hline 76 & $17_{0,17}-16_{1,16}{ }^{\star}$ & 102501.056 & 2 & 40 & 0.6 & 30 & $\begin{array}{c}\text { Blend with } \mathrm{C}_{2} \mathrm{H}_{5} \mathrm{OH}, \mathrm{CH}_{3} \mathrm{CCH}, \mathrm{CH}_{3} \mathrm{OCHO}, v_{\mathrm{t}}=1 \text {, and } \\
\qquad \mathrm{CH}_{3}{ }^{13} \mathrm{CCH}\end{array}$ \\
\hline 78 & $17_{0,17}-16_{0,16^{\star}}$ & 102501.227 & 2 & 40 & 175.5 & 30 & $\begin{array}{c}\text { Blend with } \mathrm{C}_{2} \mathrm{H}_{5} \mathrm{OH}, \mathrm{CH}_{3} \mathrm{CCH}, \mathrm{CH}_{3} \mathrm{OCHO}, v_{\mathrm{t}}=1 \text {, and } \\
\qquad \mathrm{CH}_{3}{ }^{13} \mathrm{CCH}\end{array}$ \\
\hline 80 & $15_{4,12}-14_{4,11}$ & 104246.661 & 2 & 39 & 143.8 & 48 & Blend with $\mathrm{SO}_{2}$ and $\mathrm{C}_{2} \mathrm{H}_{5} \mathrm{OCHO}$ \\
\hline 81 & $14_{4,10}-13_{4,9}$ & 104292.014 & 1 & 35 & 135.7 & 48 & Blend with ${ }^{13} \mathrm{CH}_{3} \mathrm{OH}$ and $\mathrm{CH}_{3} \mathrm{OCHO}$ \\
\hline 82 & $28_{12,16}-28_{11,17}$ & 104293.171 & 6 & 166 & 0.6 & 48 & Blend with ${ }^{13} \mathrm{CH}_{3} \mathrm{OH}$ and $\mathrm{CH}_{3} \mathrm{OCHO}$ \\
\hline 83 & $15_{12,3}-14_{12,2}{ }^{\star}$ & 104955.530 & 1 & 69 & 56.6 & 28 & Strong $\mathrm{C}_{2} \mathrm{H}_{3} \mathrm{CN}$ \\
\hline 85 & $15_{11,5}-14_{11,4}^{\star}$ & 105091.282 & 1 & 63 & 72.7 & 28 & $\begin{array}{c}\text { Group issue, blend with } \mathrm{U} \text {-line and } \mathrm{C}_{2} \mathrm{H}_{3} \mathrm{CN}, v_{11}=2 \text {, } \\
\text { uncertain baseline }\end{array}$ \\
\hline 87 & $15_{10,6}-14_{10,5^{\star}}$ & 105267.568 & 1 & 58 & 87.4 & 28 & Blend with U-line \\
\hline 89 & $15_{9,7}-14_{9,6}$ & 105505.313 & 1 & 54 & 100.7 & 37 & Candidate detected line, noisy \\
\hline 90 & $15_{9,6}-14_{9,5}$ & 105505.510 & 1 & 54 & 100.7 & 37 & Candidate detected line, noisy \\
\hline 91 & $15_{8,8}-14_{8,7}$ & 105840.034 & 1 & 50 & 112.6 & 43 & Noisy \\
\hline 92 & $15_{8,7}-14_{8,6}$ & 105845.040 & 1 & 50 & 112.6 & 43 & Noisy \\
\hline 93 & $16_{3,14}-15_{3,13}$ & 106119.548 & 2 & 42 & 157.5 & 25 & Candidate detected line, noisy \\
\hline 94 & $20_{8,13}-20_{6,14}$ & 106313.344 & 16 & 81 & 2.7 & 25 & Blend with U-line and $\mathrm{NH}_{2}{ }^{13} \mathrm{CHO}, v_{12}=1$ \\
\hline 95 & $15_{7,9}-14_{7,8}$ & 106313.995 & 1 & 47 & 123.0 & 25 & Blend with $\mathrm{U}$-line and $\mathrm{NH}_{2}{ }^{13} \mathrm{CHO}, v_{12}=1$ \\
\hline 96 & $16_{2,14}-15_{2,13}$ & 106333.641 & 2 & 42 & 157.6 & 25 & Blend with $\mathrm{C}_{2} \mathrm{H}_{5} \mathrm{CN}, v_{13}=1 / v_{21}=1$ \\
\hline 97 & $49_{16,33}-49_{15,34}$ & 106336.541 & 6 & 472 & 1.2 & 25 & Blend with $\mathrm{C}_{2} \mathrm{H}_{5} \mathrm{CN}, v_{13}=1 / v_{21}=1$ \\
\hline 98 & $15_{7,8}-14_{7,7}$ & 106398.557 & 1 & 47 & 123.0 & 25 & Blend with U-line and $\mathrm{C}_{2} \mathrm{H}_{5} \mathrm{OCHO}$ \\
\hline 99 & $15_{5,11}-14_{5,10}$ & 106503.501 & 1 & 41 & 139.1 & 25 & Blend with U-line \\
\hline 100 & $15_{3,12}-14_{3,11}$ & 106784.068 & 2 & 39 & 145.7 & 34 & Strong $\mathrm{OC}^{34} \mathrm{~S}$ \\
\hline 101 & $15_{6,10}-14_{6,9}$ & 106805.353 & 1 & 44 & 132.0 & 34 & Blend with U-line and $\mathrm{H}^{13} \mathrm{CCCN}, v_{5}=1 / v_{7}=3$ \\
\hline 102 & $58_{14,45}-57_{16,42}$ & 107248.283 & 9 & 625 & 2.0 & 24 & Strong $\mathrm{C}_{2} \mathrm{H}_{5} \mathrm{CN}, v_{13}=1 / v_{21}=1$ \\
\hline 103 & $17_{2,16}-16_{2,15}$ & 107249.573 & 2 & 44 & 171.4 & 24 & Strong $\mathrm{C}_{2} \mathrm{H}_{5} \mathrm{CN}, v_{13}=1 / v_{21}=1$ \\
\hline 104 & $17_{1,16}-16_{1,15}$ & 107254.820 & 2 & 44 & 171.4 & 24 & Strong $\mathrm{C}_{2} \mathrm{H}_{5} \mathrm{CN}, v_{13}=1 / v_{21}=1$ \\
\hline 105 & $15_{6,9}-14_{6,8}$ & 107674.692 & 2 & 44 & 132.1 & 24 & Blend with $\mathrm{C}_{2} \mathrm{H}_{5} \mathrm{CN}, v_{20}=1$ \\
\hline 106 & $36_{8,28}-36_{8,29}^{0,0}$ & 108387.475 & 5 & 241 & 24.2 & 48 & Group issue?, blend with $\mathrm{CH}_{3} \mathrm{C}(\mathrm{O}) \mathrm{CH}_{3},{ }^{13} \mathrm{CH}_{3} \mathrm{OH}$, and $\mathrm{C}_{2} \mathrm{H}_{5} \mathrm{OH}$ \\
\hline 107 & $18_{0,18}-17_{1,17}^{\star}$ ฝ & 108389.721 & 2 & 45 & 0.6 & 48 & Group issue?, blend with $\mathrm{CH}_{3} \mathrm{C}(\mathrm{O}) \mathrm{CH}_{3},{ }^{13} \mathrm{CH}_{3} \mathrm{OH}$, and $\mathrm{C}_{2} \mathrm{H}_{5} \mathrm{OH}$ \\
\hline 109 & $18_{0,18}-17_{0,17}^{\star}{ }^{\star}$ & 108389.794 & 2 & 45 & 186.0 & 48 & Group issue?, blend with $\mathrm{CH}_{3} \mathrm{C}(\mathrm{O}) \mathrm{CH}_{3},{ }^{13} \mathrm{CH}_{3} \mathrm{OH}$, and $\mathrm{C}_{2} \mathrm{H}_{5} \mathrm{OH}$ \\
\hline 111 & $16_{4,13}-15_{4,12}$ & 110490.325 & 2 & 44 & 154.3 & 32 & Blend with $\mathrm{CH}_{3} \mathrm{C}(\mathrm{O}) \mathrm{CH}_{3}$ \\
\hline 112 & $15_{5,10}-14_{5,9}$ & 110619.212 & 2 & 42 & 140.5 & 32 & Blend with $\mathrm{H}_{3} \mathrm{C}^{13} \mathrm{CN}, v_{8}=1, \mathrm{C}_{3} \mathrm{H}_{7} \mathrm{CN}$, and U-line \\
\hline 113 & $15_{4,11}-14_{4,10}$ & 111481.907 & 2 & 40 & 146.4 & 35 & Noisy \\
\hline 114 & $43_{20,24}-42_{21,22}$ 太 & 111917.941 & 9 & 406 & 0.5 & 29 & Blend with U-line \\
\hline 116 & $16_{13,3}-15_{13,2}^{\star}$ & 111918.685 & 1 & 79 & 57.0 & 29 & Blend with U-line \\
\hline 118 & $32_{13,19}-32_{12,21}$ & 111920.127 & 6 & 212 & 1.9 & 29 & Blend with U-line \\
\hline 119 & $17_{3,15}-16_{3,14}$ & 112029.767 & 2 & 47 & 167.8 & 29 & Candidate detected line, partial blend with U-line \\
\hline 120 & $16_{12,4}-15_{12,3^{\star}}$ & 112048.455 & 1 & 74 & 73.4 & 42 & Blend with $\mathrm{C}_{2} \mathrm{H}_{5} \mathrm{OH}$ and $\mathrm{C}_{2} \mathrm{H}_{5} \mathrm{CN}, v_{13}=1 / v_{21}=1$ \\
\hline 122 & $17_{2,15}-16_{2,14}$ & 112142.881 & 2 & 47 & 167.9 & 42 & Blend with ${ }^{13} \mathrm{CH}_{3} \mathrm{OH}$ \\
\hline 123 & 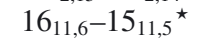 & 112212.410 & 1 & 68 & 88.5 & 42 & Blend with $\mathrm{CH}_{2}(\mathrm{OH}) \mathrm{CHO}$ \\
\hline 125 & $16_{3,13}-15_{3,12}$ & 112270.581 & 2 & 44 & 155.3 & 42 & Blend with $\mathrm{C}_{2} \mathrm{H}_{5} \mathrm{OH}$ and $\mathrm{CH}_{3} \mathrm{NH}_{2}$ \\
\hline 126 & $16_{10,7}-15_{10,6}^{\star}$ & 112426.272 & 1 & 63 & 102.3 & 31 & Group issue?, noisy, partial blend with t- $\mathrm{HCOOH}$ and $\mathrm{C}_{2} \mathrm{H}_{5} \mathrm{CN}$ \\
\hline 128 & $16_{9,8}-15_{9,7}$ & 112716.126 & 1 & 59 & 114.7 & 31 & Noisy \\
\hline 129 & $16_{9,7}-15_{9,6}$ & 112716.787 & 1 & 59 & 114.7 & 31 & Noisy \\
\hline 130 & $40_{13,27}-40_{12,29}$ & 113122.216 & 4 & 313 & 1.6 & 40 & Strong $\mathrm{CN}$ in absorption \\
\hline 131 & $11_{3,9}-10_{2,9}$ & 113123.287 & 4 & 19 & 0.8 & 40 & Strong CN in absorption \\
\hline 132 & $16_{8,9}-15_{8,8}$ & 113124.459 & 1 & 55 & 125.8 & 40 & Strong CN in absorption \\
\hline 133 & $18_{1,17}-17_{2,16}$ & 113134.125 & 2 & 49 & 0.6 & 40 & Strong $\mathrm{CN}$ in absorption \\
\hline 134 & $18_{2,17}-17_{2,16}^{2,16}$ & 113136.160 & 2 & 49 & 181.8 & 40 & Strong CN in absorption \\
\hline 135 & $16_{8,8}-15_{8,7}$ & 113138.350 & 1 & 55 & 125.8 & 40 & Strong $\mathrm{CN}$ in absorption \\
\hline 136 & $18_{1,17}-17_{1,16}$ & 113138.596 & 2 & 49 & 181.8 & 40 & Strong $\mathrm{CN}$ in absorption \\
\hline 137 & $18_{2,17}-17_{1,16}$ & 113140.632 & 2 & 49 & 0.6 & 40 & Strong $\mathrm{CN}$ in absorption \\
\hline 138 & $16_{5,12}-15_{5,11}$ & 113351.780 & 1 & 46 & 150.3 & 28 & Noisy, uncertain baseline \\
\hline 139 & $16_{7,10}-15_{7,9}$ & 113678.285 & 2 & 52 & 135.6 & 34 & Blend with ${ }^{13} \mathrm{CH}_{3} \mathrm{CH}_{2} \mathrm{CN}$, U-line, and $\mathrm{CH}_{2}(\mathrm{OH}) \mathrm{CHO}$ \\
\hline 140 & $16_{7,9}-15_{7,8}$ & 113872.664 & 2 & 52 & 135.6 & 34 & Blend with U-line and $\mathrm{C}_{2} \mathrm{H}_{5} \mathrm{OH}$ \\
\hline
\end{tabular}


Table C.1. continued.

\begin{tabular}{|c|c|c|c|c|c|c|c|}
\hline (1) & Transition $^{b}$ & $\begin{array}{c}\text { Frequency } \\
(\mathrm{MHz}) \\
(3)\end{array}$ & $\begin{array}{c}\text { Unc. }^{c} \\
(\mathrm{kHz}) \\
(4)\end{array}$ & $\begin{array}{l}E_{1}^{d} \\
(\mathrm{~K}) \\
(5)\end{array}$ & $\begin{array}{l}S \mu^{2} \\
\left(\mathrm{D}^{2}\right) \\
(6)\end{array}$ & $\begin{array}{c}\sigma^{e} \\
(\mathrm{mK}) \\
(7)\end{array}$ & Comments $^{f}$ \\
\hline 141 & $28_{3,25}-28_{3,26}{ }^{\star}$ & 114103.638 & 7 & 130 & 11.9 & 33 & Blend with $\mathrm{H}^{13} \mathrm{CC}^{13} \mathrm{CN}, \mathrm{CH}_{2} \mathrm{CH}^{13} \mathrm{CN}$, and $\mathrm{C}_{2} \mathrm{H}_{5} \mathrm{CN}, v_{13}=1 / v_{21}=1$ \\
\hline 143 & $16_{6,11}-15_{6,10}$ & 114104.283 & 2 & 49 & 144.0 & 33 & Blend with $\mathrm{H}^{13} \mathrm{CC}^{13} \mathrm{CN}, \mathrm{CH}_{2} \mathrm{CH}^{13} \mathrm{CN}$, and $\mathrm{C}_{2} \mathrm{H}_{5} \mathrm{CN}, v_{13}=1 / v_{21}=1$ \\
\hline 144 & $28_{4,25}-28_{3,26}^{\star}$ & 114104.436 & 7 & 130 & 0.3 & 33 & Blend with $\mathrm{H}^{13} \mathrm{CC}^{13} \mathrm{CN}, \mathrm{CH}_{2} \mathrm{CH}^{13} \mathrm{CN}$, and $\mathrm{C}_{2} \mathrm{H}_{5} \mathrm{CN}, v_{13}=1 / v_{21}=1$ \\
\hline 146 & $19_{0,19}-18_{1,18}^{\star}$ & 114278.164 & 2 & 50 & 0.7 & 33 & Strong $\mathrm{C}_{2} \mathrm{H}_{3} \mathrm{CN}, v_{11}=1$ \\
\hline 148 & $19_{0,19}-18_{0,18^{\star}}$ & 114278.195 & 2 & 50 & 196.5 & 33 & Strong $\mathrm{C}_{2} \mathrm{H}_{3} \mathrm{CN}, v_{11}=1$ \\
\hline 150 & $56_{14,43}-55_{16,40}$ & 115700.229 & 8 & 586 & 2.2 & 79 & Blend with $\mathrm{CH}_{2}{ }^{13} \mathrm{CHCN}$ \\
\hline 151 & $16_{6,10}-15_{6,9}$ & 115701.658 & 2 & 49 & 144.2 & 79 & Blend with $\mathrm{CH}_{2}{ }^{13} \mathrm{CHCN}$ \\
\hline 152 & $21_{5,17}-20_{5,16}$ & 145123.473 & 2 & 77 & 203.2 & 25 & Strong $\mathrm{CH}_{3} \mathrm{OH}$ \\
\hline 153 & $20_{5,15}-19_{5,14}$ & 147479.979 & 2 & 72 & 194.8 & 31 & Strong $\mathrm{CH}_{3} \mathrm{CN}, v_{8}=1$ and ${ }^{13} \mathrm{CH}_{3} \mathrm{CH}_{2} \mathrm{CN}$ \\
\hline 154 & $21_{13,9}-20_{13,8}{ }^{\star}$ & 147535.354 & 2 & 110 & 135.9 & 31 & Strong $\mathrm{CH}_{3} \mathrm{OCHO}$ and $\mathrm{CH}_{3} \mathrm{CN}, v_{8}=1$ \\
\hline 156 & $21_{12,10}-20_{12,9}{ }^{\star}$ & 147825.170 & 2 & 104 & 148.3 & 31 & Strong $\mathrm{C}_{2} \mathrm{H}_{3} \mathrm{CN}, v_{15}=1$ \\
\hline 158 & $25_{1,24}-24_{2,23}{ }^{\star}$ & 154338.749 & 2 & 93 & 0.8 & 112 & Blend with $\mathrm{NH}_{2}{ }^{13} \mathrm{CHO}$ and $\mathrm{C}_{2} \mathrm{H}_{5} \mathrm{CN}, v_{20}=1$ \\
\hline 160 & $25_{1,24}-24_{1,23}{ }^{\star}$ & 154338.764 & 2 & 93 & 254.9 & 112 & Blend with $\mathrm{NH}_{2}{ }^{13} \mathrm{CHO}$ and $\mathrm{C}_{2} \mathrm{H}_{5} \mathrm{CN}, v_{20}=1$ \\
\hline 162 & $22_{14,8}-21_{14,7}^{\star}$ & 154453.622 & 2 & 123 & 137.3 & 112 & Strong $\mathrm{CH}_{3} \mathrm{OCH}_{3}$ \\
\hline 164 & $28_{8,20}-27_{9,18}$ & 154455.607 & 9 & 148 & 0.9 & 112 & Strong $\mathrm{CH}_{3} \mathrm{OCH}_{3}$ \\
\hline 165 & $22_{13,10}-21_{13,9}^{\star}$ & 154719.395 & 2 & 117 & 150.2 & 112 & Strong $\mathrm{C}_{2} \mathrm{H}_{3} \mathrm{CN}$ \\
\hline 167 & $23_{10,14}-22_{10,13}$ & 163482.214 & 2 & 109 & 195.6 & 38 & Noisy, blend with ${ }^{13} \mathrm{CH}_{3} \mathrm{CH}_{2} \mathrm{CN}$ and $\mathrm{C}_{2} \mathrm{H}_{3} \mathrm{CN}$ \\
\hline 168 & $43_{8,35}-42_{9,33}$ & 163485.530 & 4 & 331 & 0.7 & 38 & Noisy, blend with ${ }^{13} \mathrm{CH}_{3} \mathrm{CH}_{2} \mathrm{CN}$ and $\mathrm{C}_{2} \mathrm{H}_{3} \mathrm{CN}$ \\
\hline 169 & $23_{10,13}-22_{10,12}$ & 163516.006 & 2 & 109 & 195.6 & 38 & Blend with $\mathrm{CH}_{3} \mathrm{OCHO}$ and $\mathrm{C}_{2} \mathrm{H}_{5} \mathrm{OH}$ \\
\hline 170 & $66_{17,49-66_{16,50}}$ & 163516.472 & 8 & 825 & 1.5 & 38 & Blend with $\mathrm{CH}_{3} \mathrm{OCHO}$ and $\mathrm{C}_{2} \mathrm{H}_{5} \mathrm{OH}$ \\
\hline 171 & $24_{9,15}-23_{9,14}$ & 172528.863 & 2 & 112 & 216.3 & 44 & Strong $\mathrm{HC}^{13} \mathrm{CCN}, v_{7}=1$ and $\mathrm{HC}^{13} \mathrm{CCN}, v_{6}=1$ \\
\hline 172 & $18_{4,14}-17_{3,14}$ & 176020.163 & 5 & 55 & 1.6 & 365 & Noisy, blend with $\mathrm{CH}_{2} \mathrm{CH}^{13} \mathrm{CN}$ and $\mathrm{HNCO}, v_{5}=1$ \\
\hline 173 & $71_{21,51}-70_{23,48}$ & 176022.135 & 31 & 967 & 3.4 & 365 & Noisy, blend with $\mathrm{CH}_{2} \mathrm{CH}^{13} \mathrm{CN}$ and $\mathrm{HNCO}, v_{5}=1$ \\
\hline 174 & $25_{14,12}-24_{14,11}{ }^{\star}$ & 176024.611 & 2 & 146 & 180.0 & 365 & Noisy, blend with $\mathrm{CH}_{2} \mathrm{CH}^{13} \mathrm{CN}$ and $\mathrm{HNCO}, v_{5}=1$ \\
\hline 176 & $65_{17,49}-65_{16,50}$ & 176029.258 & 6 & 794 & 1.4 & 365 & Noisy, blend with $\mathrm{CH}_{2} \mathrm{CH}^{13} \mathrm{CN}$ and $\mathrm{HNCO}, v_{5}=1$ \\
\hline 177 & $48_{20,29}-48_{19,29}$ & 176417.411 & 7 & 481 & 2.9 & 365 & Noisy, blend with $\mathrm{NH}_{2} \mathrm{CHO}$ and $\mathrm{H}^{13} \mathrm{CCCN}, v_{5}=1 / v_{7}=3$ \\
\hline 178 & $48_{20,28}-48_{19,29}$ & 176417.822 & 7 & 481 & 1.0 & 365 & Noisy, blend with $\mathrm{NH}_{2} \mathrm{CHO}$ and $\mathrm{H}^{13} \mathrm{CCCN}, v_{5}=1 / v_{7}=3$ \\
\hline 179 & $25_{13,13}-24_{13,12}$ * & 176418.067 & 2 & 140 & 191.3 & 365 & Noisy, blend with $\mathrm{NH}_{2} \mathrm{CHO}$ and $\mathrm{H}^{13} \mathrm{CCCN}, v_{5}=1 / v_{7}=3$ \\
\hline 181 & $48_{20,29}-48_{19,30}$ & 176422.486 & 7 & 481 & 1.0 & 365 & Noisy, blend with $\mathrm{NH}_{2} \mathrm{CHO}$ and $\mathrm{H}^{13} \mathrm{CCCN}, v_{5}=1 / v_{7}=3$ \\
\hline 182 & $48_{20,28}-48_{19,30}$ & 176422.897 & 7 & 481 & 2.9 & 365 & Noisy, blend with $\mathrm{NH}_{2} \mathrm{CHO}$ and $\mathrm{H}^{13} \mathrm{CCCN}, v_{5}=1 / v_{7}=3$ \\
\hline
\end{tabular}

Notes. The horizontal lines mark discontinuities in the observed frequency coverage. Only the transitions associated with a modeled line stronger than $20 \mathrm{mK}$ are listed. ${ }^{(a)}$ Numbering of the observed transitions associated with a modeled line stronger than $20 \mathrm{mK} .{ }^{(b)}$ Transitions marked with $\mathrm{a}^{\star}$ are double with a frequency difference less than $0.1 \mathrm{MHz}$. The quantum numbers of the second one are not shown. ${ }^{(c)}$ Frequency uncertainty. ${ }^{(d)}$ Lower energy level in temperature units $\left(E_{1} / k_{\mathrm{B}}\right) .{ }^{(e)}$ Calculated $\mathrm{rms}$ noise level in $T_{\mathrm{mb}}$ scale. ${ }^{(f)}$ Group means a group of transitions that are blended together. Issue means that the modeled transition or group of transitions may be inconsistent with the observed spectrum. 